\title{
The ancillary effects of nanoparticles and their implications for nanomedicine
}

\author{
Evan P. Stater', Ali Y. Sonay $\mathbb{( 1 )}^{2}$, Cassidy Hart ${ }^{3}$ and Jan Grimm ${ }^{1,2,4 凶}$
}

\begin{abstract}
Nanoparticles are often engineered as a scaffolding system to combine targeting, imaging and/or therapeutic moieties into a unitary agent. However, mostly overlooked, the nanomaterial itself interacts with biological systems exclusive of application-specific particle functionalization. This nanoparticle biointerface has been found to elicit specific biological effects, which we term 'ancillary effects'. In this Review, we describe the current state of knowledge of nanobiology gleaned from existing studies of ancillary effects with the objectives to describe the potential of nanoparticles to modulate biological effects independently of any engineered function; evaluate how these effects might be relevant for nanomedicine design and functional considerations, particularly how they might be useful to inform clinical decision-making; identify potential clinical harm that arises from adverse nanoparticle interactions with biology; and, finally, highlight the current lack of knowledge in this area as both a barrier and an incentive to the further development of nanomedicine.
\end{abstract}

rom a medical perspective, nanotechnology (in the form of nanomaterials) represents a wide frontier of novel technologies that are potentially applicable to advanced medical treatments, both for diagnostics and for therapy. Conversely, nanomaterials also are involved in a vast and (thus far) poorly mapped network of interactions with their biological environment. Nanoparticles (NPs, defined as particles $100 \mathrm{~nm}$ or less in size) used in biomedical research are highly diverse in structure, chemical composition, size, morphology, electrostatic charge, hydrophobicity, surface chemistry and other properties. A direct consequence of this diversity is an even more diverse web of biological interactions within living systems, which is rarely investigated in detail and can result in unexpected ancillary 'yin and yang' biological effects of NPs-for better or worse. Thus far, the body of knowledge that has been assembled on this subject is far from complete or consistent. Most research into NPs has been and remains goal-directed; that is, studied NPs were specifically designed to solve a problem, either as a research tool or as an investigational therapeutic or diagnostic agent. Consequently, the NP is studied primarily in terms of the problem it was intended to solve, without a comprehensive evaluation of possible ancillary interactions of the NP within its biological environment. Flexibility as molecular 'scaffolds' lends NPs to functionalization with targeting moieties, imaging agents and drugs intended to elicit a particular biological effect within a specific and assumed well-defined context. However, the non-payload portion of NPs, which frequently comprise the vast majority of the particle, is not merely inert material; it, too, interacts with surrounding biology in both overt and subtle ways. There has been comparatively little exploration of how the payload-independent composition of NPs affects and influences the biological systems with which it comes into contact, especially if the effects fall outside the scope of the NPs' intended purpose. Yet, without a thorough understanding of the possible interactions between NPs and biology, sufficient knowledge to predict a nanomedicine's safety and total effects is lacking. More extensive knowledge of nanobiology will enable us to design better NPs to overcome the limitations and problems inherent to existing NP technologies.
Furthermore, as we show here, some of the unanticipated effects of NPs in biological systems could even be exploited for novel therapeutic paradigms in nanomedicine.

Direct NP-induced toxicities (for example, cell death, oxidative stress and inflammatory responses) are the most thoroughly studied effects associated with NP exposure. However, nanomaterials may provoke more subtle effects, such as cell signalling cascades, gene expression, differentiation and cell migration, among others. The long-term implications of such effects are not known and are a potentially major barrier to the widespread translation of nanomedicines. Conversely, these biological effects, if understood, could also be exploited for medical purposes.

This Review focuses on the most relevant effects induced by clinical or translationally relevant NPs in the context of nanotherapy to inform future NP use and design, and also to provide a framework to address the core issues that are holding the field back. As in vitro data from NP studies have not been found to robustly correlate with in vivo observations, we focus primarily on literature that describes ancillary effects with supporting results obtained in vivo. We will also interpret the potential importance of these findings for the future of medicine and public health and underline the need to improve our understanding of the non-directed biological effects of nanomaterials.

The structure of nanoparticles and biological 'identity' Nanoparticle classification and 'pristine identity'. A particular type of NP is distinguished from others by its unique physical and chemical characteristics. The most obvious method to categorize an NP is based on the physicochemical composition of the core material; this is the primary component of the NP identity. However, although the core constitutes a major part of the particle, the first contact with the particle's environment is made not by the core but through the NP's outer coating. Therefore, NPs are often defined secondarily by the outward-facing surface of the particle, which frequently is of a different material to that in the central portion. This classification method works well for uniform or 'core-shell'-type

'Department of Pharmacology, Weill Cornell Medicine, New York, NY, USA. ${ }^{2}$ Molecular Pharmacology Program, Memorial Sloan Kettering Cancer Center, New York, NY, USA. ${ }^{3}$ Department of General Surgery, Lankenau Medical Center, Wynnewood, PA, USA. ${ }^{4}$ Department of Radiology, Memorial Sloan Kettering Cancer Center, New York, NY, USA.凶e-mail: grimmj@mskcc.org 
nanostructures, prevalent in biomedical research, in which the core can be either organic or inorganic, with the outer shell or coating mostly organic for a greater biocompatibility and solubility. There are, of course, highly complex nanostructures, which defy classification under this simplistic methodology. NPs are sometimes also descriptively defined by other physicochemical traits: shape or geometry (tube, rod, sphere, star and so on), surface charge (neutral, anionic or cationic), surface chemistry functional groups (for example, amine, carboxyl and thiol), porosity, hydrophobicity, rigidity and so on. These physicochemical traits define the foundational 'pristine' identity of the particle and drive its interactions with biology via its affinity to various types of biomolecules.

Nanoparticle corona and 'biological identity'. Before direct interaction with the cellular surface or internal cellular machinery of particular organ systems, NPs will typically come into contact with extracellular fluids (ECFs) such as blood, lymph and interstitial fluid, which contain a broad range of (bio)molecules. Owing to the typically high surface-to-mass ratio of nanoscale structures, extensive interaction with these biomolecules occurs ${ }^{1}$ and forms an affiliated biomolecule shell, or NP 'corona', which includes mineral ions, lipids, sugars, carbohydrates and, especially, proteins ${ }^{2}$. The composition of these coronas is determined by the biological environment and the pristine identity of the particle. NP coronas are complex, heterogeneous and dynamic, and are formed almost instantaneously by a process that is spontaneous, stochastic and irreversible ${ }^{3}$. Furthermore, the composition of the corona is temporally dynamic and contextually dependent; the corona composition shortly after the initial ECF exposure may differ from that of the corona of an NP immersed in that same ECF for hours ${ }^{4}$. The type of ECF in which the NP is dispersed, variations in the ECF content due to biological differences (for example, species, sex and age), the initial ECF composition at the route of NP entry, the tissue and/or organ context ${ }^{5}$, disease states ${ }^{6}$ and NP metabolism and/or decay (among many other conditions) can all result in differing corona compositions. Abundant molecules at the initial entry side (usually the blood, that is, serum) typically constitute most of the corona.

The corona appears to serve a dual purpose: it naturally functions to mitigate NP toxicity by 'walling off' the foreign material from delicate biological structures with a protective barrier of biomolecules ${ }^{7}$, and simultaneously facilitates the clearance of the NP by scavenging immune cells. However, the biomolecules that associate with the nanomaterial are important mediators of many complex biological processes whose functions could conceivably be impacted by interaction with NPs. The primary interface of NPs with complex living systems is typically indirect, via engagement with NP-associated corona components, rather than a direct interaction with the nanomaterial ${ }^{8}$. For this reason, the corona make-up and assembly dynamics are a critical factor in directing NP-biological interactions ${ }^{2,8}$. The corona thus constitutes a bioactive shell that can bind to and activate cell surface receptors and other biological mechanisms based on the corona composition and NP localization ${ }^{3,9-11}$. It has been shown that immunoglobulins and lipoproteins found in serum can engage their respective receptors when adsorbed into silica NP coronas; the receptor binding of the protein epitopes remain accessible even when bound to the $\mathrm{NP}^{12}$. The bioactivity of coronas can both drive NP uptake by binding to endocytic receptors and activate other signalling cascades; it can also interact with the extracellular matrix or the cell membrane. The pristine identity of the NP may also redefine the corona component functionality by altering the secondary, tertiary or quaternary structure of the adsorbed corona proteins through particle-protein interaction $^{3,13,14}$. Corona effects are probably not limited to interactions with extracellular or cell surface features, as coronas have been shown to persist after uptake, and therefore could continue to mediate the interface with intracellular biology even after endocytosis, which leads to various intracellular effects ${ }^{7}$.

The coronal association of biomolecules, driven by environmental context and NP pristine identity, could impart unanticipated biological activity on the particle-defining an NP's 'biological identity' on top of its pristine identity and driving ancillary effects through interactions. Importantly, the corona is not the sole determinant of NP-mediated biological effects, and a corona does not necessarily abrogate other effects that may arise from interactions with the NP core material or other non-coronal components ${ }^{15,16}$. As with much of our understanding of NP-biological interactions, our understanding of NP coronas (and the resulting impacts on the surrounding biology) is fragmented and incomplete. Understanding of the relationship between pristine identity and biological identity, and the interaction of particle biological identity with biological systems, is crucial for the further development of nanomedicines.

\section{Ancillary effects of nanomedicines, a double-edged sword}

Traditionally, NPs were viewed as excipients of drugs. However, the diverse assortment of payload-independent NP effects represents a potential untapped avenue of biological mechanisms which, in an appropriate context, might be leveraged for medicinal benefit. That is, rather than merely functioning as a passive excipient, an NP might itself exhibit drug-like effects. These ancillary effects could conceivably be exploited either independently as a stand-alone nanomedicine or coupled with a conventional small molecule or biological drug to improve and enhance the therapeutic efficacy. However, properties of nanomaterials can also induce potentially harmful effects at the cellular, tissue and organ system levels with detrimental consequences for patients who receive the nanomedicine (Fig. 1). The potential of certain nanomaterials to trigger inflammation and cytotoxicity is well-known ${ }^{1}$; however, other ancillary effects can be more subtle and might have pernicious effects. This Review considers selected relevant findings of potential medical usages for clinically relevant NPs (or preclinical NPs with translational potential), as well as some notable health and safety concerns that arise from the use of NPs as a medical tool, based on the intrinsic effects arising from NP-biological interaction-the yin and yang of nanomedicine, interconnected intimately.

Effects on vasculature and biological barriers. The usefulness of drugs is highly dependent on their potential to reach the site of intended action. For example, a drug that is highly effective in vitro when directly applied to cells is therapeutically useless if it cannot reach those cells in vivo. Thus, biological barriers effectively function as tissue and organ gatekeepers, and dictate which tissues are accessible to which drugs. The foremost barrier that affects pharmaceuticals is the blood vasculature, as most agents must diffuse through it to reach the site of intended action. In particular, the blood-brain barrier (BBB) is a major challenge for drug development, as it is less permissive and more selective to drugs than are other vessels, and allows almost no paracellular diffusion ${ }^{17}$. It is known that nanomaterial characteristics, particularly particle size and shape, can influence the degree to which NPs can pass through vascular barriers ${ }^{18}$. However, certain translationally relevant NPs (notably gold, titanium dioxide and silica) exhibit ancillary effects that modulate the permeability vascular endothelial barriers. NPs could therefore be utilized as agents to facilitate or improve drug delivery at sites by modulating the permissiveness of the barrier to those tissues, which are otherwise problematic to reach for biological or small-molecule therapies. This permeabilization, however, could present unforeseen problems by dysregulating the protective functions of the BBB and other vasculature, and potentially expose the brain or other vulnerable organs to toxins, pathogens or metastatic cells that would otherwise be excluded by an intact barrier. 


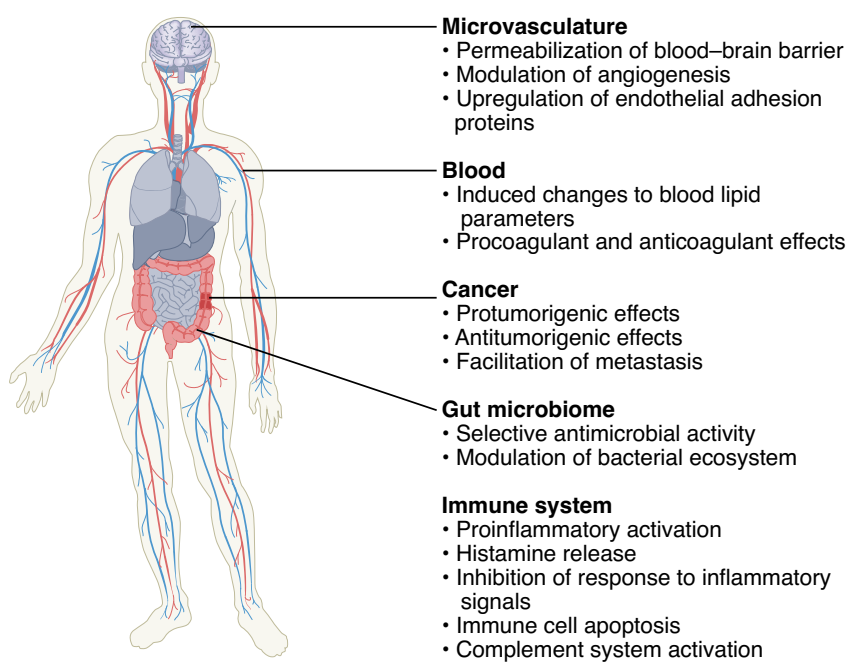

Fig. 1 | Summary of select biological effects associated with translationally relevant nanomaterial. Translationally relevant nanoparticles can cause a diverse assortment of ancillary effects in a wide variety of organs and tissues. This diagram categorizes observed nanoparticle effects described in this review. The nature of some of these effects are potentially relevant to human health and medicine, as they may cause patient harm or impact therapeutic efficacy. Such ancillary activities must be taken into consideration in the design of future nanomedicines. Redrawn from image owned by Evan Paul Stater and Jan Grimm and created by Ella Marushchenko, Springer Nature Ltd.

The integrity of microvasculature is sustained by cell-cell adhesion between vascular endothelial cells, which forms a tight 'seal' that prevents uncontrolled extravasation. Various types of NPs have been shown to affect the integrity of the blood vasculature in several organs, which include the $\mathrm{BBB}$, in ways that can even drive metastasis. Certain NPs can induce the formation of micrometre-scale pores in the vascular endothelium, which create leaks through which dysregulated paracellular diffusion can occur. For example, in microvasculature, adherens junctions initiate and maintain cellcell adhesion in the vascular endothelium using cell-cell adhesion proteins called cadherins and catenins. The interactions between the domains of cadherins in neighbouring cells initiates the formation of an adherens junction. Titanium dioxide NPs were shown to bind to vascular endothelial cadherin (VE-cadherin) and disrupt the interactions between the VE-cadherin molecules that form the adherens junctions, which increases the bidirectional permeability of the vascular endothelium in subcutaneous blood vessels and in healthy lung vasculature ${ }^{19,20}$. Disruption of interendothelial vascular junctions was also observed with citrate-coated silver NPs, which triggers VE-cadherin downregulation. Gold NPs were found to increase the degradation rates of tight junction proteins in the $\mathrm{BBB}^{21}$. Therefore, NP ancillary effects on vasculature could both compromise the integrity of the vasculature or create opportunities to deliver drugs beyond otherwise highly selective barriers.

In addition to the ancillary effects on vascular integrity, NPs might also influence the biological processes that regulate neovascularization. Zinc oxide NPs were found to enhance the development of capillary formation both in vitro and in vivo. The metallic component of the NP catalyses the formation of intracellular reactive oxygen species (ROS) in vascular endothelial cells, which including hydrogen peroxide, a redox-active second messenger in the angiogenic signalling process. This subsequently drives sprouting and the formation of new blood vessels from the existing microvasculature ${ }^{22}$. Such an NP may function to rapidly improve circulation at an injury site, which facilitates further tissue growth and repair. Therefore, stimulation of the neovascularization by an NP could be beneficial in enhancing healing. NP-based tissue engineering approaches are particularly interesting for the frontiers of regenerative medicine, and include bone tissue regeneration, treatment of myocardial infarct injuries, scar tissue repair, cosmetic surgery and organ transplantation. It is plausible that these emerging medical technologies could benefit by incorporating NPs to harness the ancillary effects for an improved efficacy and effects otherwise potentially difficult to achieve.

Haemostatic regulation and alteration of blood parameters by NPs. NPs can modulate mechanisms that regulate the delicate balance behind haemostasis and haemostatic regulation, in both procoagulant and anticoagulant directions. Blood clotting is a complex process, typically initiated by the adhesion of platelets at a site of vascular injury, followed by an activation cascade of soluble clotting factors, which generate an insoluble network of cross-linked fibrin proteins. Some types of NPs were shown to affect platelet adhesion, the initial step. Silver NPs, either uncoated ${ }^{23}$ or PEGylated (PEG, polyethylene glycol $)^{24}$, reduce platelet adhesion by altering the conformation of the adhesion protein integrin $\alpha_{\mathrm{II}} \beta_{3}$, which has anticoagulant effects in mice by ultimately reducing the downstream generation of thrombin and fibrin. Similarly sized uncoated gold NPs have no comparable antiplatelet effects ${ }^{24}$. The effects on haemostatic regulation can also be mediated by NP interaction with secreted clotting factors. In this context, cationic poly(amidoamine) dendrimers acted as a procoagulant by promoting the formation of fibrinogen aggregates via a thrombin-independent mechanism, in which the cationic dendrimer interacts directly with fibrinogen ${ }^{25}$. The clot-forming potential of erythrocytes can be affected by NPs as well, as titanium dioxide NPs upregulate phosphatidylserine exposure on erythrocytes, which facilitates thrombin formation and erythrocyte aggregation in rats $^{26}$. Furthermore, NPs might modulate endothelial cell adhesiveness: silica NPs were observed to upregulate endothelial PECAM (platelet endothelial cell adhesion molecule) expression and increase platelet adhesion to the vascular wall, which could contribute to thrombus formation ${ }^{27}$. It is unlikely that nanotherapies would function more effectively than the existing drugs for systemic haemostatic regulation; however, these effects could be exploited for localized haemostatic effects consistent with another therapeutic objective, for example, by enhancing the uptake of NPs into tumours by inhibiting intratumorally platelet activation $^{28}$. However, these effects are potentially also undesirable off-target effects and could contribute to risks such as brain ischaemia or cardiac infarction. NP therapies thus might be contraindicated in patients receiving anticoagulants.

NPs can potentially affect the formation and pathology of atherosclerotic plaques. In atherosclerosis, fatty lesions develop along the wall of blood vessels, which impairs blood flow and ultimately increases the risk of ischaemia and acute infarct or stoke. These lesions are typically inflamed due to infiltration by macrophages, which become laden with phagocytosed lipids, such as low-density lipoprotein, and promote disease progression. Long-term inhalation exposure to titanium dioxide ${ }^{29}$ and zinc oxide ${ }^{30} \mathrm{NPs}$, which are commonly found in many consumer goods, has been shown to induce blood dyslipidaemia in rodents by an unknown mechanism. These changes, which include the elevation of low-density lipoprotein and total cholesterol, and the elevation of serum triglycerides with a subsequent increased risk of atherosclerosis and plaque formation. Further, risks could arise from NPs meddling with inflammatory functions, as above in atherosclerotic plaques, and both possibly enhance their formation or slow their progress.

Microbiotic regulation. There are relatively few identified therapeutic applications for orally administered nanomedicines. However, NPs are already abundant in consumer products, which 
include sunscreens, toothpaste, cosmetics, topical medications and food additives. The commonality of nanomaterial in these products means that exposure via the oral and inhalation routes is unavoidable and largely unknown. The microbial ecosystem of the gastrointestinal tract, the gut microbiome, is a highly complex and dynamic biological milieu; current research is revealing an ever-expanding effect of the gut microbiome on multiple systems. Resident microbiota can be beneficial, commensal or pathogenic to the host; thus, upsetting the delicate balance of this ecosystem might impair digestive efficiency or leave the host more vulnerable to opportunistic infections. The gut microbiome has recently been implicated in the modulation or manipulation of an unexpectedly wide array of host biological processes, which include metabolism, immunoregulation and even behaviour. Although a mechanistic understanding of the specific interactions between microbes and host remains poorly understood, NPs may also interact with bacteria or other microorganisms in the microbiome, and thus potentially impact the poorly understood interactions between host and microorganism ${ }^{31}$. From a product safety and public health perspective, the effects of internalized NPs on the microbiota of these organs, and their broader health consequences, remains largely an unresolved question.

Silver and silver-composition NPs possess broad antimicrobial activity and are frequently incorporated into topical medical treatments for burns as an anti-infective agent. However, different species and strains of bacteria exhibit varying degrees of sensitivity to silver toxicity ${ }^{32}$. Thus, silver NPs affect certain constituents of the bacterial species commonly found in the gastrointestinal tract more than in others: the oral intake of silver NPs alters the microbiome homeostasis in mice and rats $^{33-36}$. Gut biome perturbations are not exclusive to silver NPs; exposures to gold nanoclusters ${ }^{37}$, titanium dioxide $\mathrm{NPs}^{36}$ and zinc oxide $\mathrm{NPs}^{38}$ have also demonstrated the alteration of gut homeostasis in mice. In addition to bacteria, bacteriophages are an integral component of the microbiome, and represent a phage virome that interacts with and modulates the gut bacterial ecology. The phage virome composition might also be impacted by NP exposure; for example, the long-term intake of silver NPs by rhesus monkeys was shown to reduce the gut population of bacteriophages known to prey on pathogenic bacteria ${ }^{39}$.

The gut microbiome is also only one of many identified microbiomes in the human body. Though poorly studied, the lung microbiome is believed to have roles in the regulation of host immunity and homeostasis similarly to the gut microbiome and is also easily accessible to inhaled external nanomaterials. Microbicidal silver NPs disrupt the composition of the mouse lung microbiome in a coating-dependent manner, with citrate-coated silver NPs being the most disruptive ${ }^{40}$. To make matters even more complex, NPs could also exert effects on tumour microbiomes, the relevance of which to cancer has only recently come into focus ${ }^{41-44}$.

The current limited understanding of the relationship between microbiome and host biology constrains our ability to interpret the relevance of these NP-mediated perturbations of microbial populations, or to determine whether the induced changes might be beneficial, detrimental or inconsequential. However, some ancillary effects on the host were observed in a few of the aforementioned studies. For example, rats fed with silver NPs exhibited changes in behavioural tests and histopathological alterations in the brain, although it is unclear whether the NP-induced microbiome disruption was a causative factor ${ }^{34}$. In another rat study, silver-NP-induced microbiome changes were accompanied by a decrease in expression of the immunomodulatory genes in intestinal tissue, which suggests a modulation of the host immune response $\mathrm{e}^{33}$.

Immunomodulation. NPs exhibit a wide range of effects on the immune system, even in translationally relevant forms. NP-mediated immunomodulation can be pro- or anti-inflammatory and downstream may affect other activities not directly related to inflammation, such as tissue remodelling or scavenging functions, as well as immune cell differentiation, maturation and senescence. To emphasize the variability of ancillary effects that arise from NPbiological interactions, we highlight notable studies that illustrate the divergent immunomodulatory effects of NPs on unwanted systemic immunomodulation. The elicited ancillary effects are highly dependent on the physicochemical properties of the NPs, the immune cell subset interacting with NPs and the nature of the surrounding environment.

Immunostimulation and pro-inflammatory effects. The potential of many nanoscale materials to induce inflammation in organs and tissues is probably one of the earliest observed effects of nanobio interactions. It is highly likely that ancillary mechanisms are integral to NP-mediated inflammatory responses. NP-triggered inflammatory-response mechanisms are highly diverse and complex, but generally can be categorized into one of three mechanistic schemes: the intracellular route, wherein NPs are taken up by sentinel immunocytes or other cells and provoke inflammation through various intracellular mechanisms; the Fc (fragment crystallizable)-mediated immune response route, wherein humoral immunogenicity of the nanomaterial results in extensive opsonization with NP-specific antibodies and subsequent Fc receptor activation; and the complement system activation route, caused by the extracellular interaction of complement immunity factors with nanomaterials. With respect to the intracellular pathway of inflammation activation, there are any number of mechanisms by which cellular binding or the uptake of nanomaterial could provoke inflammation. Such possibilities are too complex and varied to discuss in detail in this Review, and are extensively covered elsewhere ${ }^{1}$; however, such complexity is illustrative of the diversity of NP-mediated effects in biology. Generally, an inflammatory response will be driven by the release of inflammatory mediators (tumour necrosis factor, interleukins, interferons, ATP and so on) from cells exposed to nanomaterial. Such a response could be provoked in any number of known (or unknown) ways: for example, NP uptake leads to endosomal build-up and leakage followed by NP contact with cellular machinery, which causes varied responses, including protein unfolding and interference with cell signalling cascades, disruption of cellular metabolism and increased oxidative stress, and activation of damage-associated molecular pattern receptors and autophagic pathways. These effects and others, all ultimately, lead to the release of inflammatory mediators by secretion or by cell lysis, which triggers recruitment and activation of the surrounding immune system and paracrine inflammatory responses in neighbouring cells. Importantly, the nature and magnitude of such effects are highly dependent on NP identity (both pristine and biological) and biological context. The activation of the complement system by NPs is another important ancillary effect that is also a recurring clinical problem with approved nanodrugs in current use, and therefore is described in greater detail further on in this section.

NP-induced immune activation, generally considered an unwanted side effect, could be medically useful in certain circumstances: for example, to combat infection, to provoke a host defence response against cancer or to stimulate the antigenicity of vaccines. The pro-inflammatory nature of many nanomaterials may therefore be useful as a supplemental adjuvant for vaccines. Indeed, microparticle formulations of aluminium compounds (for example, Alhydrogel) have been in clinical use in adjuvanted vaccines for decades (and gave rise to false and unwarranted but ongoing controversies). Investigations of nanoscale formulations of aluminium showed an improved adjuvant activity compared with those of microscale forms, as well as a reduced induction of local inflammation ${ }^{45}$. This improvement was attributed to the increased potency of nanoalum in NLRP3 inflammasome activation in antigen-presenting cells (APCs) ${ }^{46}$. Conventional microscale 
aluminium-based adjuvants are known to elicit a strong $\mathrm{T}$ helper cell $2\left(\mathrm{~T}_{\mathrm{H}} 2\right)$-type immune response by $\mathrm{CD}^{+}$helper $\mathrm{T}$ cells. Although efficient in provoking a strong and durable humoral immune response to antigens by B cells, they lack a strong induction of a $\mathrm{T}_{\mathrm{H}} 1$-type helper $\mathrm{T}$ cell immune response and thus are not particularly effective at stimulating persistent cell-mediated immunity by the activation of $\mathrm{CD}^{+} \mathrm{T}$ cells. Thus, their utility is limited in vaccines against diseases for which antibody responses are not particularly effective (for example, the human immunodeficiency virus). However, nanoscale aluminium particles may be able to circumvent this deficiency. A polyacrylic acid-stabilized nanoscale formulation of Alhydrogel elicited a potent $\mathrm{T}_{\mathrm{H}} 1$ immune response in a murine model $^{47}$, which suggests that NP adjuvant formulations could be used to appropriately tune the adaptive immune response in vaccinations for which a stronger cell-mediated immunity is required. Additionally, other nanomaterials were found to exhibit strong adjuvant activity with a minimal local inflammation, such as biocompatible lipid NP formulations ${ }^{48-50}$, which could provide a promising adjuvant alternative to the irritation-producing aluminium salts or Toll-like receptor agonist adjuvants, and most recently used in COVID-19 vaccines. Immunostimulatory ancillary effects could also conceivably be useful in cancer; such effects and applications are discussed in the 'Ancillary effects in cancer and cancer therapy' section below.

Complement activation. In addition to leukocytes, the complement system is affected by NPs as well. Complement is a biochemical defence that comprises secreted factors, which act in parallel to and in conjunction with the other arms of the immune system. The complement system is driven by the interaction of complement factors with biochemically foreign surfaces and has three main functions: direct lysis of foreign cells (the 'classical pathway'), opsonization of foreign cells and debris to facilitate clearance (the 'alternative pathway') and induction of inflammation to attract phagocytes (the 'lectin pathway'). Complement immunity factors are often found within NP coronas; interaction with the NP surface or with other corona biomolecules can trigger activation of complement factors, which potentially activate any of the three complement pathways ${ }^{51}$. However, activation of the alternative pathway appears to be the most prevalent and best-characterized effect of NP interaction with this system ${ }^{52,53}$. Alternative pathway activation by dextran-coated iron oxide NPs has been closely studied. The initial step in this process appears to be the adsorption of complement factor $\mathrm{C} 3$ onto the NP corona. The resultant molecular interaction between $\mathrm{C} 3$ and other molecules on the NP surface can induce spontaneous hydrolysis of $\mathrm{C} 3$ into two subunits, $\mathrm{C} 3 \mathrm{a}$ and $\mathrm{C} 3 \mathrm{~b}$. C3b within the NP corona can further drive complement activation by forming an enzyme complex on the NP surface with other complement factors; this complex (AP convertase) catalyses the cleavage of intact C3 into $\mathrm{C} 3 \mathrm{a}$ and $\mathrm{C} 3 \mathrm{~b}$, as well as the cleavage of the complement factor $\mathrm{C} 5$ into $\mathrm{C} 5 \mathrm{a}$ and $\mathrm{C} 5 \mathrm{~b}$. The cleavage products of $\mathrm{C} 3$ and $\mathrm{C} 5$ can activate complement factor receptors in many tissues, and potentially induce a cascade of biological responses (Fig. 2). C3a and C5a are also known anaphylatoxins that elevate the risk of severe immune reactions. The potential of iron oxide NPs to activate the alternative pathway appears to be independent of the dextran structure, functionalization and dextran/iron molar ratio, which suggests that the corona is the primary driver of C3 cleavage ${ }^{54}$.

Complement activation is especially problematic for nanomedical purposes, as it can contribute to suboptimal NP pharmacokinetics and biodistribution, inflammation, allergy and potentially a serious anaphylaxis-like condition, CARPA (complement activation-related pseudoallergy), which has been observed with several clinical nanomedicines. The complement system can be activated even by highly biocompatible NPs, such as poly(lactic-co-glycolic acid) (PLGA) ${ }^{55}$, carbohydrate-coated iron oxide $\mathrm{NP}^{56}$ or liposomes; ${ }^{57}$ with respect to the last of these, increased activation was associated with a high negative surface charge and morphological deviation from sphericity. CARPA-like patient reactions have occurred with several clinical nanomedicines, including Doxil (PEG-liposomal doxorubicin) ${ }^{58}$ and Feraheme (ferumoxytol) ${ }^{59}$, which received an FDA black box warning as a result.

Although generally considered undesirable, NP complement activation could conceivably be leveraged for therapeutic benefit as well. When co-administered with a model antigen, rod-shaped PEG NPs promoted the alternative pathway of complement activation, which provided an adjuvant-like boost to the immune response and improved humoral immunogenicity ${ }^{60}$. Chitosan NPs have also exhibited adjuvanticity via the activation of complement and were investigated extensively ${ }^{61-63}$. Such particles could form the basis of vaccine nanoadjuvants free of metals and endotoxin derivatives.

Immunomodulation, immunosuppression and anti-inflammatory effects. We now understand that NPs can be immunoregulatory or immunosuppressive in addition to being inducers of inflammation ${ }^{64}$. Immunosuppressive characteristics of NPs have been observed in interactions with multiple immune cell classes. Such effects can include immune cell cytotoxicity or senescence, decreased antigen presentation, diminished or altered cytokine release profiles and attenuated reactivity to pathogen-associated molecular signals.

Nanomaterial immunosuppression and anti-inflammation have potential applications, especially in the context of injurious immune overactivation. Clinical immunomodulatory agents, such as corticosteroids and mTOR inhibitors, are often used to mitigate localized pathological inflammatory responses; however, these drugs have broad off-site and off-target systemic effects, which result in a complicated side-effect profile. Even without targeting, NPs are frequently preferentially incorporated into monocytes, macrophages and other myeloid-derived cells with immunoregulatory functions, and thus represent a potential passive targeting tactic for nanomaterials with immunomodulatory properties. For example, negatively charged particles composed of the biodegradable PLGA copolymer, a nanomaterial, have shown the capability to exhibit immunomodulatory effects in inflammatory monocytes. In particular, PLGA particles exhibited therapeutically beneficial anti-inflammatory and immunosuppressive effects in multiple disease models. PLGA NPs passively target inflammatory monocytes that express the scavenger receptor MARCO (macrophage receptor with collagenous structure), which promotes splenic sequestration and apoptosis and results in a depletion of these cells from distal sites of inflammation. PLGA particles were shown to act therapeutically as an anti-inflammatory agent in murine models of West Nile virus-induced encephalitis, experimental autoimmune encephalomyelitis, cardiac infarction, kidney reperfusion injury and inflammatory bowel disease ${ }^{65}$. These effects were achieved without apparent systemic immunodeficiency, a common and potentially severe side effect of anti-inflammatory drugs.

Additionally, internalized PLGA nanomaterial can suppress co-stimulatory signals from APCs, such as macrophages and dendritic cells. The mechanism behind these PLGA-mediated ancillary effects in the immune system is not well understood, but appears to be dependent on acidification caused by degradation of the particle into lactic acid and glycolic acid; therefore, it is probably a product of the pristine, rather than the biological, identity of the $\mathrm{NP}^{66}$. Highly promising applications for NPs with immunosuppressive properties (and perhaps one of the most promising for nanomedicines to date) are in the development of tolerogenic NPs for the treatment of allergy and autoimmune disease, and in minimizing rejection in human organ transplantation without systemic immune suppression. By exposing an APC to an antigen under immunosuppressive conditions, it is possible to teach the adaptive immune system to 'forget' a pathological adaptive immune response, as the 


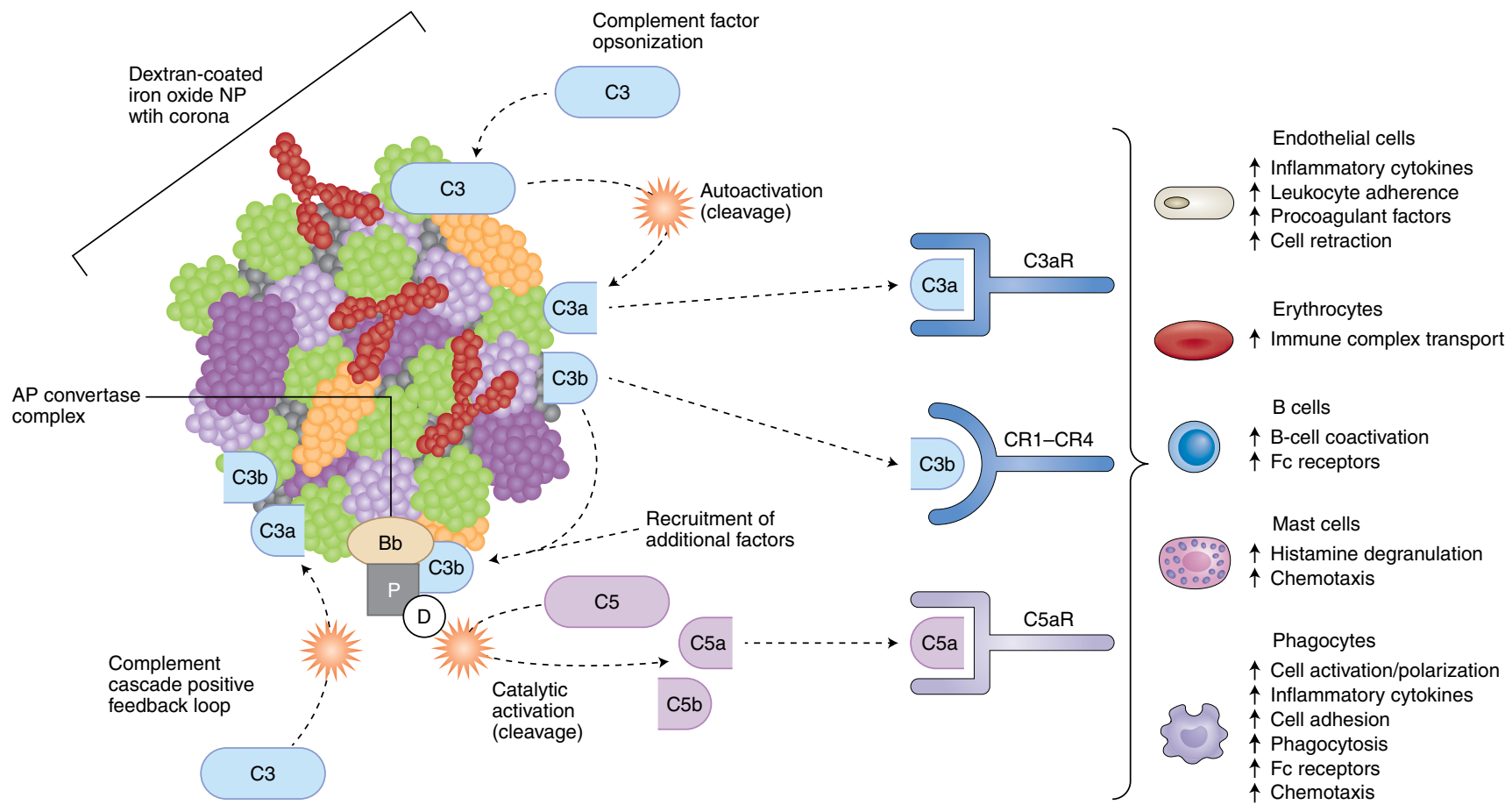

Fig. 2 Activation of the alternative pathway of the complement immune system by dextran-coated iron oxide NPs. Complement factor C3 is absorbed onto the particle surface, where interactions with the nanomaterial may trigger self-cleavage into factors C3a and C3b. Factor C3b can facilitate the formation of the alternative pathway (AP) convertase complex, which enzymatically cleaves complement factor C5 into factors C5a and C5b and creates a positive feedback activation of additional factor C3. Cleaved complement factors can function as anaphylatoxins and opsonins and activate complement factor receptors in a wide variety of cell types. Figure created with BioRender.com.

antigen is presented to $\mathrm{T}$ cells in the absence of a co-stimulatory signal, which results in ablation of antigen-reactive $\mathrm{T}$ cells ${ }^{67}$ (Fig. 3). This requires coupling an antigen to an immunosuppressive delivery NP; the delivery of the antigen to an APC (with the activation suppressed by the internalized NP) can cause the reactivity to a known antigen to be diminished or eliminated. Separate administration of the inhibitory agent has not been found to be effective; co-delivery of both the antigen and immunosuppressive agent as a unified entity appears to be required. Although the co-packaging of an mTOR inhibitor or other immunosuppressive drug payload is probably the most clinically practical configuration for a tolerogenic NP, the induction of tolerance has been demonstrated using only NPs with immunosuppressive qualities. Antigen-conjugated PLGA NPs have been shown to tolerize allergic airway inflammation in murine model ${ }^{68-70}$. Furthermore, PLGA NPs decorated with donor peptides reduced allograft rejection in several murine transplant models, which includes in skin $^{71}$ and pancreatic islet cell ${ }^{72}$ transplants, a strategy that could be an alternative to broadly immunosuppressive antirejection drugs, such as sirolimus or tacrolimus.

Ancillary effects in cancer and cancer therapy. NP effects are especially interesting in the context of cancer, because prototypic nanomedicines are frequently designed as potential cancer therapeutics (usually as a drug delivery system and often coupled with targeting and/or imaging modalities). However, the ancillary effects of the NP carrier itself on cancer biology are often not considered. The biology of cancer is atypical, dysregulated and extremely heterogeneous, and therefore presents a unique environment for NP-mediated ancillary effects. Furthermore, the uptake of NPs into tumours is often amplified due to a poorly defined vascular phenomenon described as the enhanced permeation and retention (EPR) effect, wherein the biodistribution of administered NPs is increased in tumours ${ }^{73}$. Thus, biomedical NPs infused into to a patient are likely to be deposited into the tumour microenvironment at an elevated concentration (although the magnitude of this effect is highly variable and often less pronounced in humans $)^{74}$. Therefore, we must consider the interaction of the NP with biology in a cancer setting. Here we highlight instances in which ancillary effects were observed that may have translational relevance in cancer therapies, or instances in which they may facilitate cancer growth and progression (Fig. 4).

Notably, some NPs may exhibit carcinogenicity by the induction of cellular changes that promote cancer development in normal tissues. This is a well-known property of nanomaterials with limited clinical relevance, such as carbon nanotubes, which have been shown to persist in the lungs of rodents after inhalation, and eventually cause mesothelioma or mesothelioma-like lung injury ${ }^{75-78}$. However, studies indicate a potential mutagenicity or genotoxicity from commonly used NPs, such as titanium dioxide NPs, which are nearly ubiquitous in consumer products. Although there is no conclusive evidence of a direct causal effect in carcinogenesis via typical exposure routes and at reasonable exposure levels and durations, titanium dioxide (inclusive of nanoscale forms) has recently been classified as a Group 2B substance by the International Agency for Research on Cancer, which recognizes its possible carcinogenicity ${ }^{79}$.

The immune cell compartment of tumours plays an important role in cancer development, as many cancer types can effectively 'reprogramme' or suppress infiltrating leukocytes to facilitate growth and metastasis. However, the modulation of immune cell responses is also an ancillary NP effect that could have interplay with tumour-mediated immunomodulation, and opsonized NPs are readily scavenged by the macrophages, neutrophils, dendritic cells and monocytes typically present in the tumour microenvironment. Consequential NP ancillary effects on the immune cell compartment within a tumour, and subsequent effects on the tumour itself, have so far been mostly overlooked, but there is increasing evidence that NP-mediated ancillary effects can, indeed, 


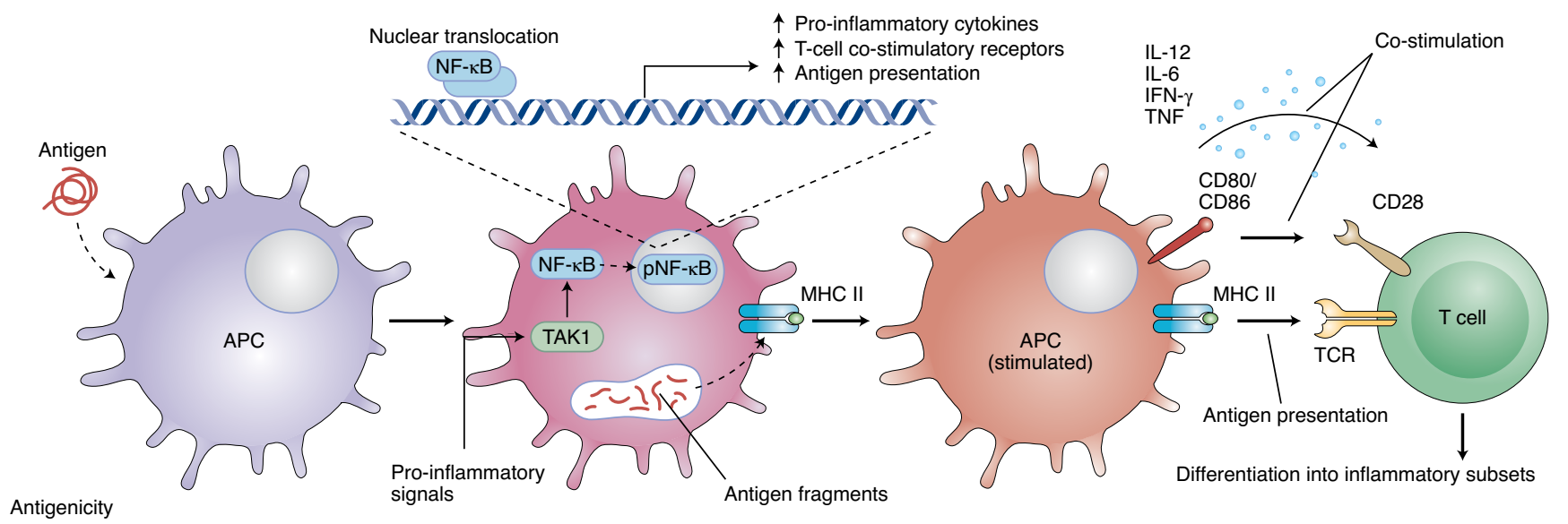

Antigenicity

signals

Tolerogenicity

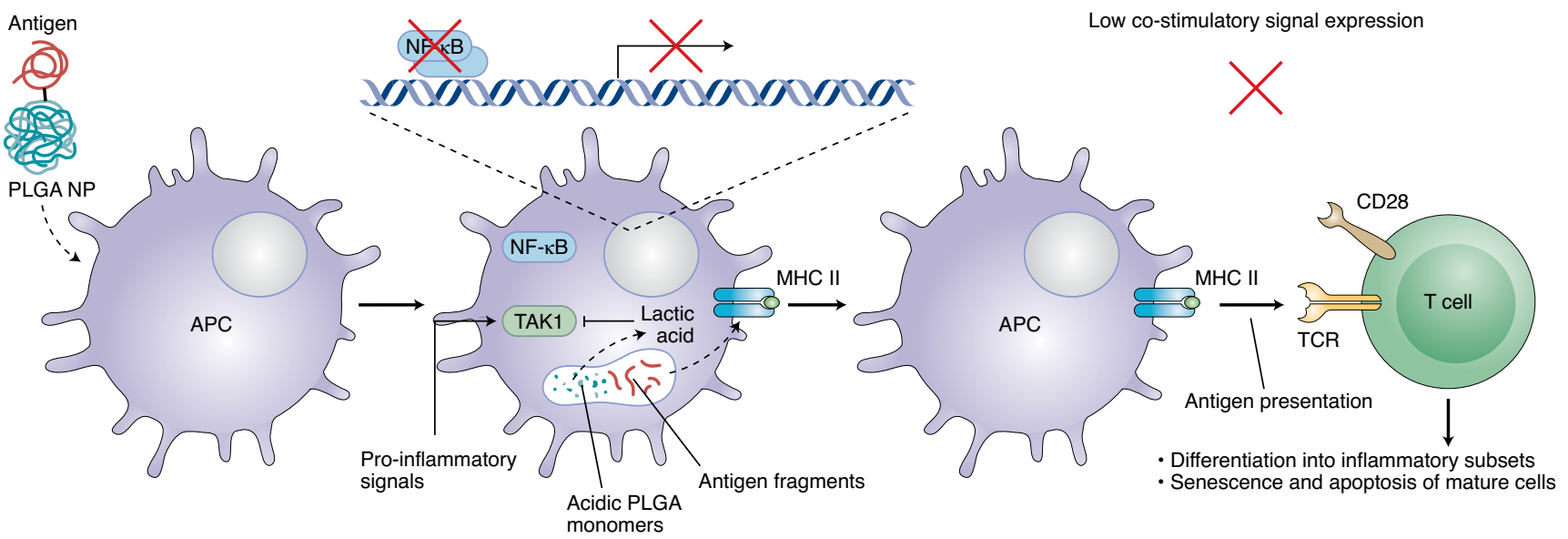

Fig. 3 | NP-mediated immunomodulatory effects contribute to antigen tolerance. Top: uptake of a foreign antigen by APCs in conjunction with extrinsic pro-inflammatory signal activation. This co-stimulation contributes to the education of naive T cells and the activation and/or expansion of mature antigen-reactive $T$ cells by an increased antigen-fragment presentation via major histocompatibility complexes, juxtacrine co-stimulatory receptor expression and secretion of pro-inflammatory cytokines. Bottom: co-uptake of antigen with PLGA nanomaterial. Degradation of PLGA into acidic monomers results in the inhibition of TAK1 (transforming growth factor- $\beta$-activated kinase 1 ) by lactic acid, which reduces phosphorylation of NF- $K B$ (NF, nuclear factor) and nuclear translocation of pNF-KB (phosphorylated NF-KB) and so blunts responsiveness to pro-inflammatory signals that signal through TAK1. Antigen-fragment presentation to $T$ cells in the absence of a modicum of co-stimulation results in the non-activation of naive cells and senescence or apoptosis of mature antigen-reactive T cells. IFN- $\gamma$, interferon- $\gamma$; IL, interleukin; MHC, major histocompatibility complex; TCT, T-cell receptor; TNF, tumour necrosis factor. Figure created with BioRender.com.

impact the surrounding cancer biology. Ferumoxytol, a clinical carboxymethyldextran-coated iron oxide NP, has shown promise as an agent to activate macrophages and counteract tumour-mediated manipulation of tumour-associated macrophages (TAM) phenotype. High-dose ferumoxytol repolarized TAM and liver Kupffer cells to an M1-like pro-inflammatory, cancer-inhibiting phenotype in a murine breast cancer-liver metastasis model; this slowed tumour growth and shielded the liver from metastatic seeding ${ }^{80}$. TAM repolarization is believed to occur through cellular iron enrichment. Abundant intracellular iron acts as a Fenton chemistry catalyst, which generates superoxide radicals and drives the adoption of the M1 phenotype (Fig. 5a). Thus, this effect is primarily elicited by the composition of the particle (pristine identity), whereas the passive targeting of the particle to TAM is driven largely by the biomolecule corona of ferumoxytol (biological identity).

Myeloid-derived suppressor cells (MDSCs), an immunosuppressive type of immature monocyte that often resides within tumours, are activated by gold NPs functionalized with a biomimetic high-density-lipoprotein-like surface via the binding and activation of SCARB1 (scavenger receptor class B type 1), which pushes myeloid cells out of the pathological MDSC phenotypic niche. This shift reduced the tumour growth in a lung cancer model, and both metastasis and growth in a melanoma model. The reduction in the tumour MDSC burden also resulted in an increase in active tumour-infiltrating cytotoxic T lymphocytes, which presumably increased the immune surveillance of the tumours ${ }^{81}$. These effects are interesting notably because lipoproteins are a common constituent of NP coronas. High-density apolipoproteins do not directly bind SCARB1; however, cholesteryl esters contained within high-density lipoprotein will specifically bind and activate the receptor, which raises the possibility that other NPs could elicit this effect as well. As this gold NP was not synthesized with cholesteryl esters, the biological activity of the particle that activates SCARB1 is probably derived from sterol esters incorporated into the corona from serum.

On the opposing end of the immune response spectrum, NPs may also modulate tumour immune cells in ways that aid cancer progression. Studies of unloaded liposomes that lack a drug payload are uncommon; in one rare study, it was shown that the liposome structure itself might have an adverse effect on cancer through 


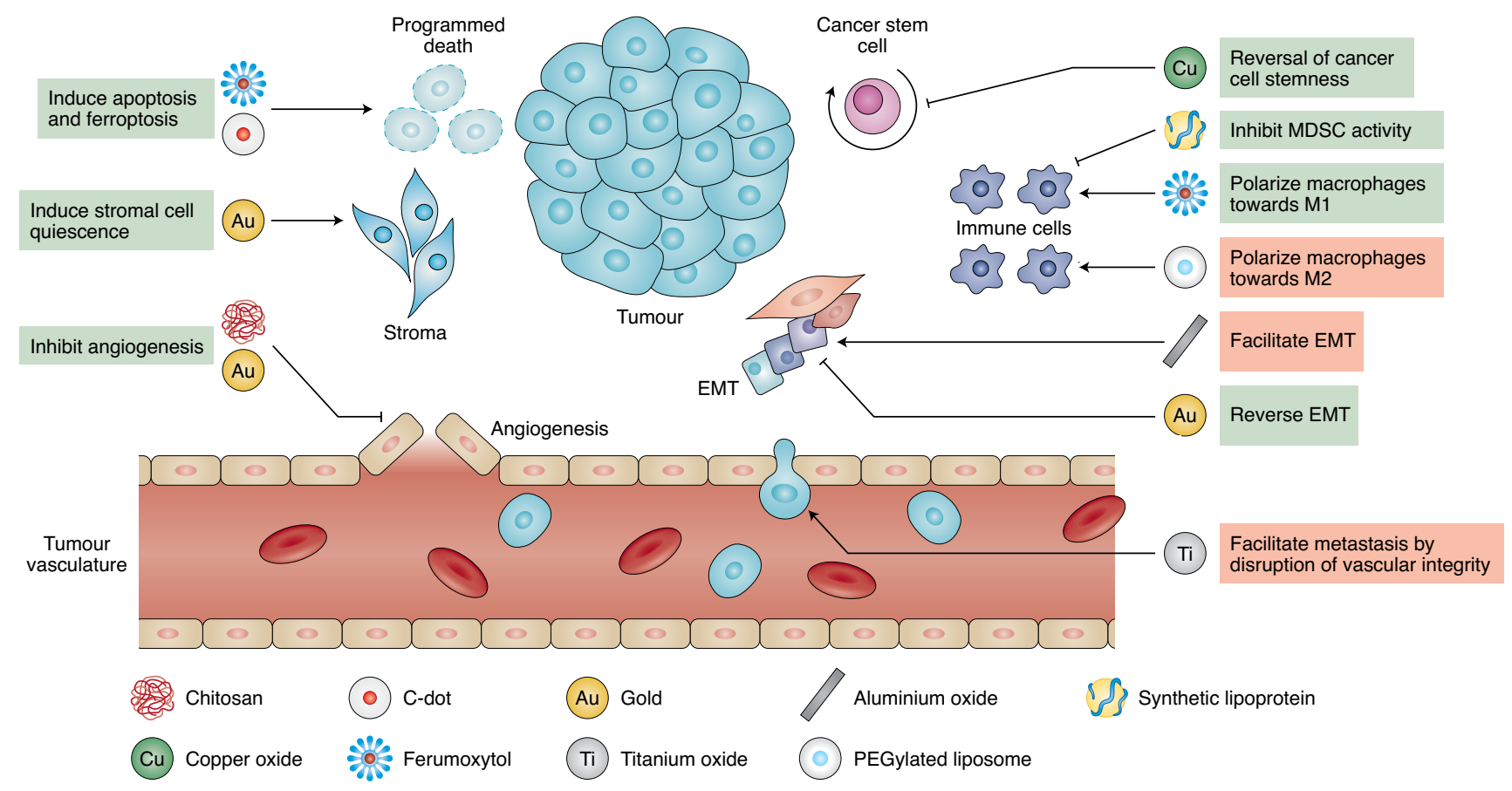

Fig. 4 | Summary of NP effects in cancer environments. Various NPs can directly affect cancer cells or tumour stroma, modulate responses of tumour-infiltrating immune cells and facilitate or inhibit metastasis and tumour angiogenesis. NP-associated effects which are likely to inhibit or facilitate cancer progression are labelled above in green or red, respectively. Redrawn from image owned by Evan Paul Stater and Jan Grimm and created by Ella Marushchenko, Springer Nature Ltd.

interactions with immune cells ${ }^{82}$. PEGylated liposomes without a drug cargo elicited a tumour-promoting M2-like phenotype in TAM in a papillomavirus-induced tumour model, which resulted in a higher secretion of $\mathrm{T}_{\mathrm{H}} 2$ cytokines and local immunosuppression in the tumour microenvironment. Treatment with unloaded liposomes consequently increased the tumour growth rate ${ }^{82}$. Thus, consideration must be given to the NP composition for drug-delivery nanopores intended for cancer therapy, as the NPs themselves might provoke responses that are counterproductive to the therapeutic mechanism.

Outside the immune cell compartment, other types of tumoral stroma cells are susceptible to antitumorigenic effects mediated by nanobiological interaction, albeit indirectly. In the absence of any other drug payload or concomitant therapy, $20 \mathrm{~nm}$ gold NPs reduce growth and metastasis of pancreatic ductal adenocarcinoma in mice. Although the effect mechanism is not immediately clear, analysis of the secretome of pancreatic cancer cells demonstrated a marked downregulation of many progrowth factors and cytokines. Correspondingly, there was a downstream reduction in extracellular matrix secretion by stromal pancreatic stellate cells, both of which reduce cancer aggressiveness ${ }^{83}$. Gold NP monotherapy has also shown efficacy in rodent models of ovarian cancer, as it reduces both tumour proliferation and metastasis by interruption of the epithelial-to-mesenchymal transition (EMT) of cancer cells, possibly by inhibition of mitogen-activated protein kinase signalling through an unknown mechanism ${ }^{84}$. In both models, gold NPs were not cytotoxic to non-cancerous cells, which suggests that the effect was due to a specific interaction of the NP or NP-associated biomolecules with the dysregulated biology of cancer cells.

Furthermore, NPs may directly contribute to programmed cell death pathway activation in cancer cells. Recently, our group showed that iron-containing NPs can modulate an iron-dependent cell death pathway called ferroptosis (Fig. 5b). The overload of ferroportin-deficient acute myeloid leukaemia cells with iron via ferumoxytol uptake generates large amounts of ROS by Fenton chemistry, which causes a build up of oxidized lipids and so triggers ferroptotic cell death in the context of low ferroportin expres$\operatorname{sion}^{15}$. Similarly, fluorescent silica carbon-quantum dots (C-dots) functionalized with a melanoma targeting peptide also induce ferroptosis in melanoma under cysteine-deficient conditions (Fig. 5d). Remarkably, pristine C-dots contain no iron; however, C-dots apparently accumulate iron from the biological environment, perhaps incorporating metallic ions into the outer silica layer or the corona $^{16}$. Therefore, proferroptotic NPs might be useful as a novel therapeutic for cancers with cellular iron export defects or be used in combination with another inducer of oxidative stress.

Perhaps more consequentially, vascular integrity perturbation by NPs might actually facilitate metastasis. The effects described by Peng et al. wherein titanium dioxide NPs disrupted VE-cadherin binding and induced leakage in the lung vasculature by (described in 'Effects on vasculature and biological barriers') were also applicable to the tumour vasculature; the increase in vascular permeability was shown to facilitate both extravasation of the tumour cells from the primary tumour and to promote the 'seeding' of metastatic sites by an increased permissiveness of the circulating tumour cells in the normal vasculature (Fig. $5 \mathrm{c})^{19}$. Further evidence suggests that this effect is not unique to titanium dioxide, and could occur with gold, silver and silica NPs as well ${ }^{19}$. An additional study determined that PEGylated gold NPs of various sizes are predominantly taken up into tumours via the transcellular route through active transport, and not via the passive paracellular diffusion process commonly attributed to EPR; these internalized NP conceivably could exert ancillary effects on endothelial cell biology from within ${ }^{85}$. Both of these findings are potentially concerning for cancer nanotherapy development, as the EPR effect may not be a completely passive process; diffusing NPs might actually influence or alter the vascular endothelium through which they pass. However, this effect might be therapeutically exploitable to facilitate the uptake of other NPs or drugs out of the blood compartment, but must be carefully balanced against a possible increased metastasis. It remains to be seen 
a

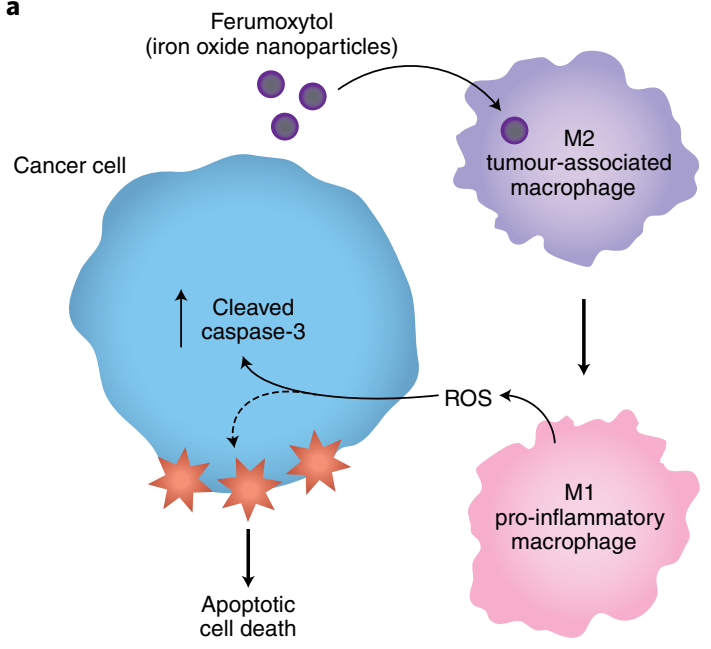

b
Leukaemic cell

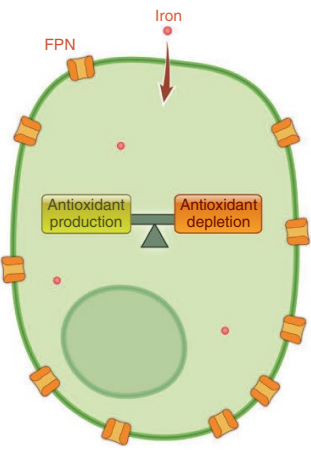

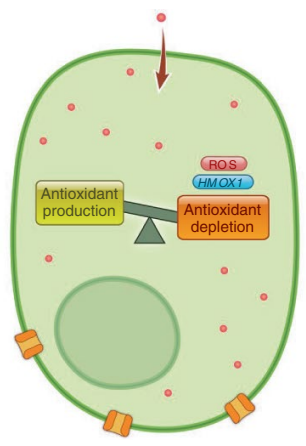

Leukaemic cell with ferumoxytol therapy

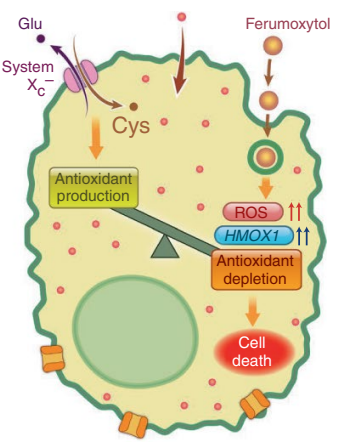

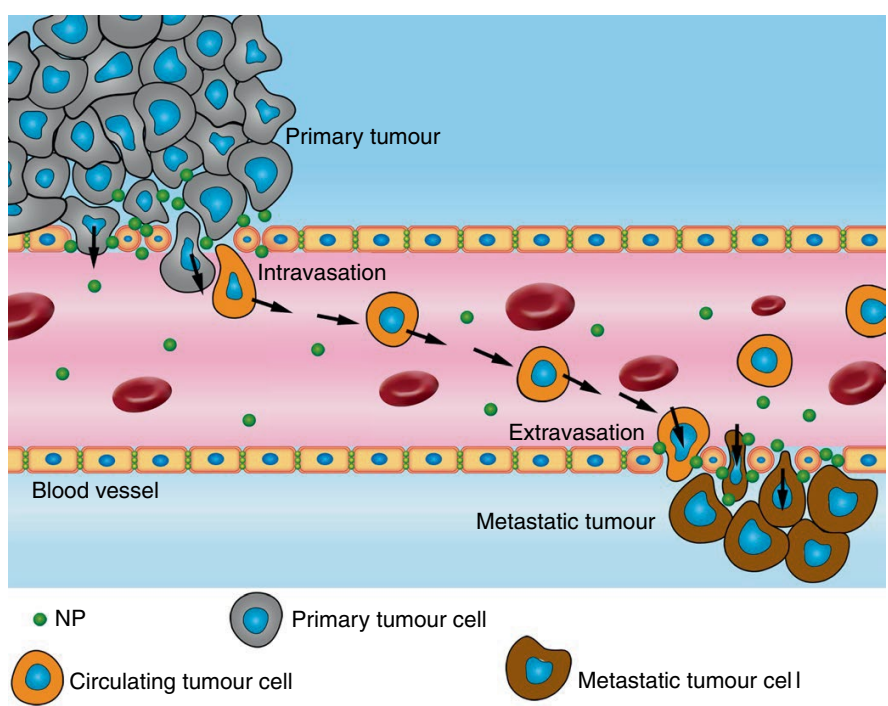

d

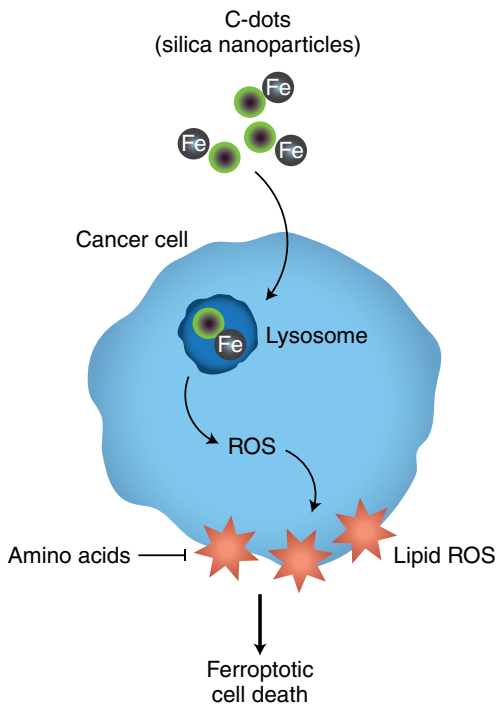

Fig. 5 | Mechanisms of action of select nanoparticle-induced ancillary effects in cancer. a, Ferumoxytol iron oxide NPs modulate the macrophage polarization in tumours to drive M1 polarization and induce killing of cancer cells via extracellular ROS generation. $\mathbf{b}$, Ferumoxytol uptake by iron-retaining leukaemic cells activates the ferroptotic pathway, which triggers cell death. c, Titanium dioxide NPs modulate endothelial cell permissiveness in microvasculature and facilitate cancer metastasis. d, C-dots accumulate iron content in vivo, which triggers ferroptotic cell death in cancer cells. FPN, ferroportin. Panels a and $\mathbf{d}$ adapted with permission from ref. ${ }^{121}$, Springer Nature Ltd. Panels reproduced with permission from: $\mathbf{b}$, ref. ${ }^{15}$, Springer Nature Ltd; c, ref. ${ }^{19}$, Springer Nature Ltd.

which NP characteristics mediate this effect, and whether this effect is recapitulated by NPs of other classes.

\section{Strategies to mitigate nanoparticle adversities}

Much effort has been focused on creating translational NP forms that are amenable to medical applications. The focus of these efforts has largely been to ameliorate obvious adverse NP effects, such as cytotoxicity, particle aggregation in ECF, inflammation, immune cell sequestration, undesirable organ targeting and/or overwhelming of the mononuclear phagocytic system ${ }^{1}$. Progress has been made in this area by gaining a better understanding of the relationship between NP characteristics and biological responses in vivo, and we are learning to better manage these effects by modulating particle properties. However, we must further identify strategies to mitigate more subtle adverse events that arise from ancillary effects. The variations in NP structure and composition, coupled with the intricate complexity of biological systems, make the prediction and control of ancillary effects challenging. NP development can follow several strategies that could lead to a better scientific understanding of NP interactions in vivo, and so allow the development of novel therapeutic approaches.

Rapid degradation and/or elimination strategies. A considerable obstacle for NPs as medicines is the vastly different routes of metabolism that separate them from small-molecule drugs or biologicals. Although the pharmacokinetic behaviour of these conventional drugs is well understood and established, NPs typically behave quite differently for reasons that are probably nested in their macromolecular scale. Foremost, a major roadblock to the translation of many nanomaterials is biopersistence. Whereas most small organic molecules are predictably and efficiently eliminated by cytochrome P450s and other metabolic pathways, many nanomaterials defy a rapid and efficient clearance from the body, because human biology lacks the metabolic mechanisms to process many nanomaterials or nanoscale structures. Also problematic is the propensity of many NPs to target certain organs, particularly 
the liver and spleen; disproportionately high local concentrations of nanomaterial there makes the elicitation of (potentially harmful) ancillary effects more likely, especially when biopersistent material lingers long after administration ${ }^{1}$. These characteristics could cause protracted off-target, off-site ancillary effects.

Constructing biomedical NPs from biodegradable nanomaterials is a reasonable and practical approach to reduce the ancillary effects that arise from biopersistence. Biodegradable material can be broken down under physiological conditions into products that are non-toxic and/or can be eliminated readily by metabolic or elimination pathways (Fig. 6a). For example, the PLGA copolymer degrades into its monomers, lactic acid and glycolic acid, both of which are natural metabolites of human physiology (although a rapid degradation of PLGA does produce transient local acidification). In addition to PLGA, which is well studied and has been in medical use for decades, several other biodegradable nanomaterials are in widespread use today, which include polylactic acid, poly(amidoamine) and chitosan ${ }^{86}$. These polymer systems are highly flexible and adaptable, and can be used to construct NPs of different sizes and shapes. Surface functionalization can also be employed to modulate the degradation rates, or to make degradation selective to a particular condition (for example, $\mathrm{pH}$ ). However, note that biodegradable nanomaterials are not necessarily biocompatible, and nanoscale materials are prone to decay in vivo by burst degradation rather than by a gradual surface erosion, which could be problematic for biodegradable NPs in medical applications ${ }^{1}$.

An alternative elimination strategy for intravenously administered NPs involves producing ultrasmall NPs that are less than approximately $10 \mathrm{~nm}$ in size with zwitterionic or positive surface charges ${ }^{87}$. Ultrasmall NPs are rapidly filtered through the kidney glomerulus and eliminated from the body in urine. Given the short duration of NPs in the system, unwanted ancillary effects can be minimized, and clinical translation of otherwise biopersistent NPs could be implemented with less concern for a long-term retention in the body ${ }^{88}$. This strategy was clinically investigated for inorganic silica-coated C-dots, which are approximately $7 \mathrm{~nm}$ in diameter and cleared renally ${ }^{89,90}$. A limitation of ultrasmall particles is the extremely rapid renal clearance from the blood; the short half-life pharmacokinetics of such a small particle may not be compatible with the therapeutic purpose of a particular nanomedicine. However, this approach could also be facilitated with biodegradable nanomaterials, for example, a larger polymeric NP that degrades or dissociates in circulation into sub-10-nm fragments that are able to undergo renal elimination.

Harnessing the corona architecture. The corona is one of the primary determinants of an NP's interactions with host biology. To date, corona formation has mostly been regarded as undesirable 'biofouling' that should be minimized, for example, via low-adhesion NP coatings, such as PEG or other 'stealth' materials, to cloak the particle from immune recognition and minimize its biological engagement. However, this strategy affects the biological fate of the NPs, and it is unlikely that corona formation can ever be completely abolished. A more forward-thinking approach to nanotherapeutics is to view the
NP-corona complex from a holistic perspective. That is, the corona should be seen as an extension of the NP, rather than as a hindrance to it, and the corona formation and composition (and how it will interface with biological systems) should be considered with respect to the particle's intended function. Essentially, we could adapt the pristine identity of the NP as the foundation on which to build the biological identity. Adjustment of the corona architecture could, therefore, be exploited to mitigate problems with nanotherapeutics, which include cytotoxicity, immunogenicity, pharmacokinetics, biodistribution, cell-specific uptake and intracellular compartmentalization-and even for the regulation of ancillary effects.

Corona pre-formation is a basic strategy wherein NPs are pre-incubated ex vivo in a defined medium of the preferred corona components prior to administration (Fig. 6b). Corona preformation allows some limited control over the corona composition, and is applicable to any existing NP type. This technique has been studied in animal models, although mostly using only albumin as the sole constituent of the preformed corona; even this relatively simple and unsophisticated method has shown the potential to reduce the binding of opsonins and complement immunity factors, as well as to improve the NP stability, pharmacokinetics and cellular uptake, and decrease the particle cytotoxicity ${ }^{91-94}$. With a greater knowledge of nano-bio interactions, more sophisticated predefined coronas could be designed with more advanced purposes, such as to facilitate NP targeting, elicit specific ancillary effects to synergize with a therapeutic function or minimize off-target ancillary effects associated with the NP nanobiology. However, this method also has limitations, as the NP corona is not static but rather is a dynamically evolving shell ${ }^{5,95}$. A preformed corona could be influential in setting an NP's initial biological identity, but it is still heterogeneously transient and subject to modification by the biological environments within the host. The host biology is likely to 'rewrite' the particle identity away from the initially defined one, although the kinetics of biomolecule exchange may still allow for practical modifications of the NP behaviour.

Another more advanced strategy for harnessing the corona is to apply an in silico modelling strategy for a rational particle design to predict and direct corona formation in vivo. Molecular dynamics and structure-activity relationship modelling software have been used to predict the corona assembly and NP-cell association ${ }^{96-98}$, and further modelling could analyse how corona-bearing NPs would interact with biological barriers, cells, tissues and organs, and predict the biological consequences. These strategies could be integrated into a rational particle design, that is, an NP's physicochemical characteristics, morphology and functionalization would be purposefully manufactured to recruit a corona of a desired composition that is compatible with the biomedical function of the NP (Fig. 6c). In effect, the pristine identity of the NP would be defined to facilitate the adoption of a specific biological identity in vivo. A few simple proof-of-concept studies have been performed with this aim. For example, the polysaccharide chain structure on the exterior of a dextran NP can be manipulated to activate specific complement immunity pathways ${ }^{99}$. The NP surface can be functionalized to recruit specific factors into the corona; an in silico strategy was

Fig. 6 | Strategies for mitigating nanoparticle adversities, which may arise from ancillary effects. a, Left: persistent NPs resist degradation and accumulate in target organs, which results in long-term biological effects. Right: biodegradation strategy enables the rapid elimination of the nanomaterial or metabolism into bioidentical building blocks. b, Top: NPs designed without consideration of the corona composition may have undesirable biological interactions, extensive opsonization, rapid clearance and/or accumulation in off-site organs and cell types. $\Delta$ t indicates the passage of an interval of time. Middle: the pre-addition of a defined corona prior to administration provides a foundation to influence further corona formation in vivo. Bottom: rational particle design strategy uses in silico modelling strategies to predict the corona structure and composition in vivo, which enables control of the particle's subsequent biological identity. c, Strategies to define the biological identity of particles by mimicking the host biology. Upper left: NP surfaces are densely functionalized with serum proteins or other native biomolecules. Upper right: NPs are conjugated to the host cell surfaces or cell-membrane-derived vesicles. Lower left: cell membranes derived from the host cells are used to completely envelop NPs within an extracellular vesicle-like structure. Lower right: biomimetic NPs utilize bioidentical materials for the particle construction. Figure created with BioRender.com. 
used to design a gold NP functionalization motif that would incorporate transferrin from blood serum into the corona. This particle exhibited a high transferrin receptor binding ${ }^{100}$. NP surface chemistry has further shown the capability to influence the conformation of bound corona components: a recent study observed that polymer NPs with different surface functional groups could stabilize or denature corona albumin proteins; such conformational differences were associated with differential binding to various types of macrophage scavenger receptors, and consequently allowed the de facto targeting of specific macrophage subsets ${ }^{101}$.

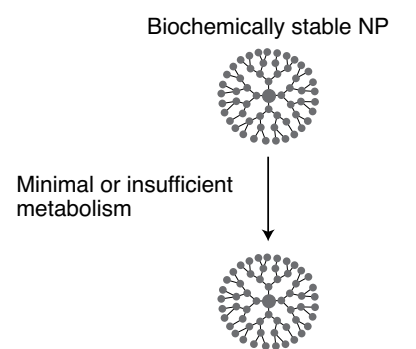

Biopersistent nanomaterial

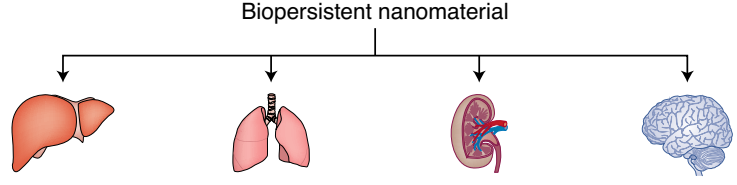

- Efficient elimination not possible

- Retention in organs of uptake

- Possible inflammation and fibrosis

- Long-term exposure to off-target ancillary effects
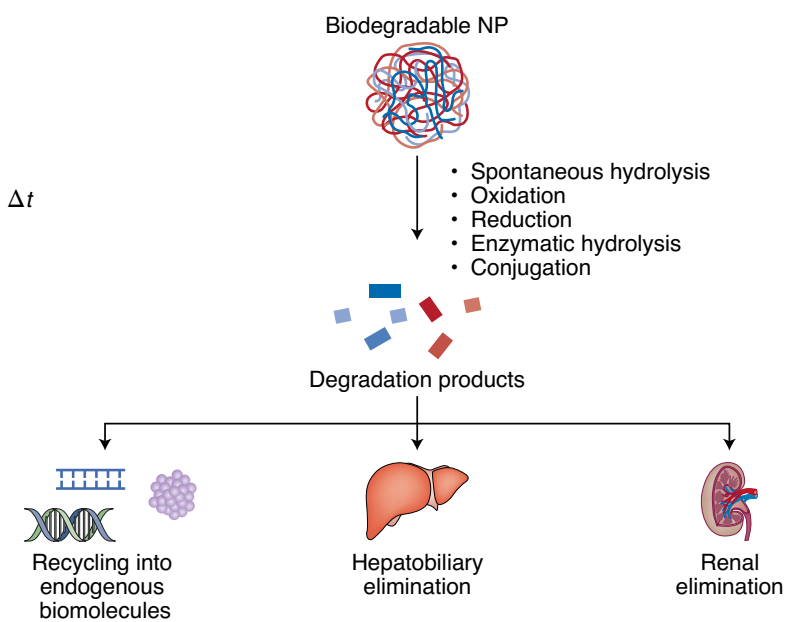

b
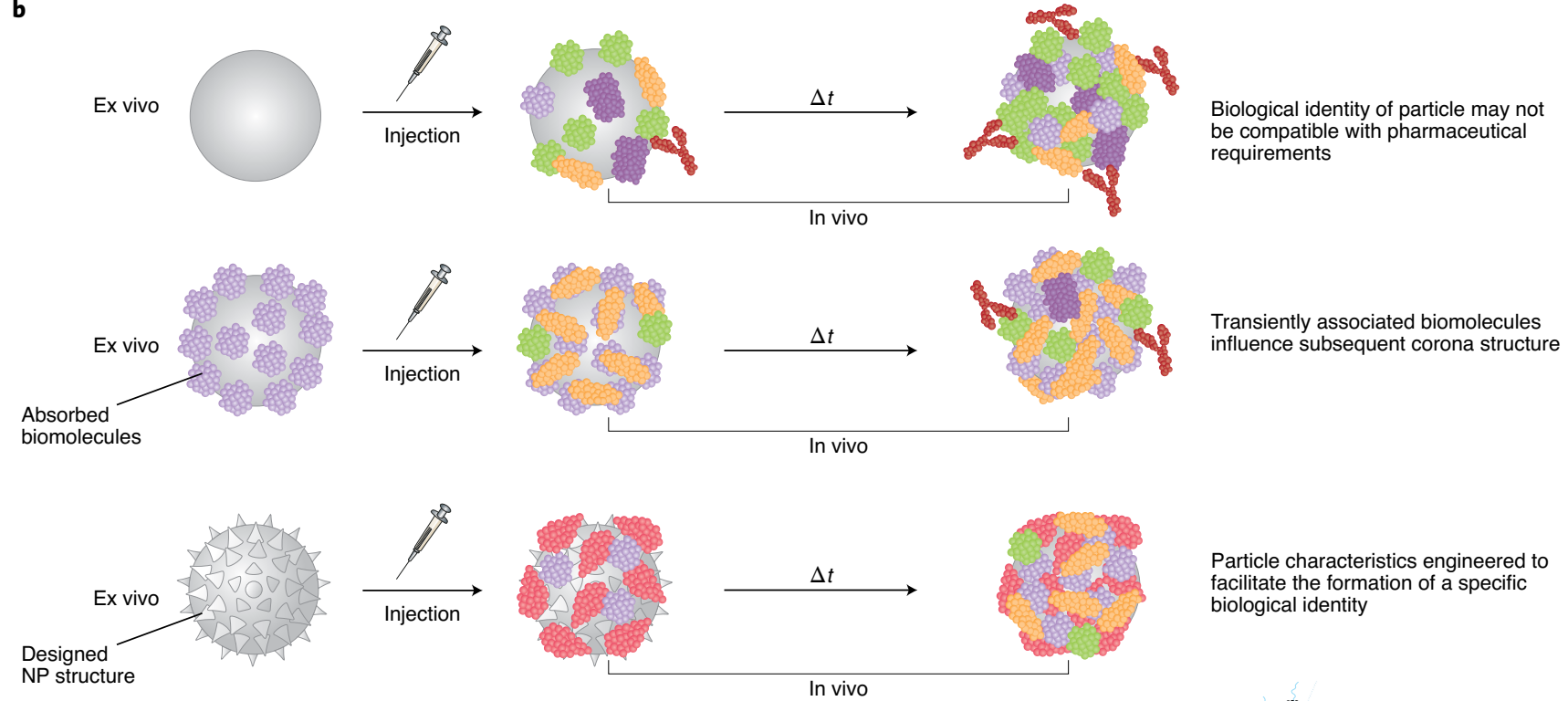

Particle characteristics engineered to facilitate the formation of a specific biological identity
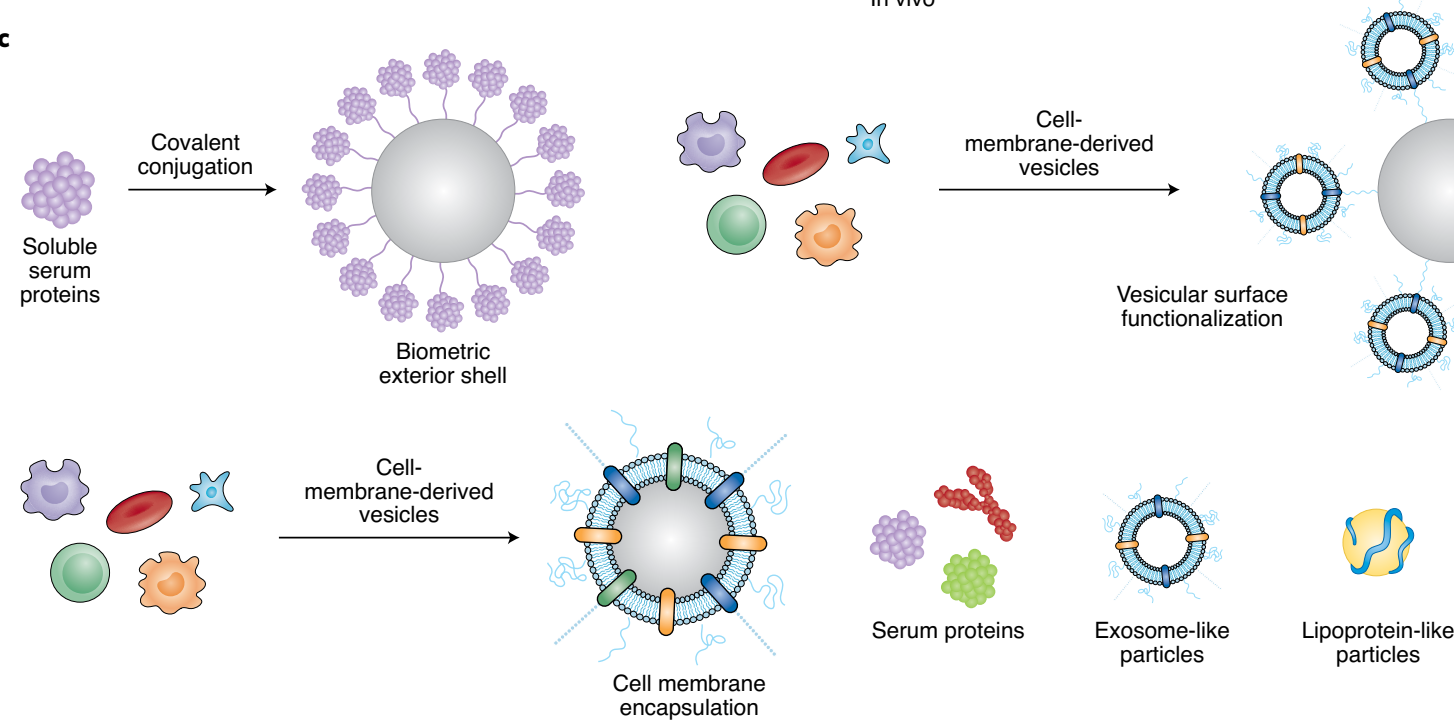

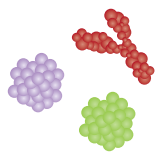

Serum proteins

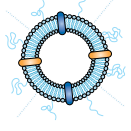

Exosome-like particles
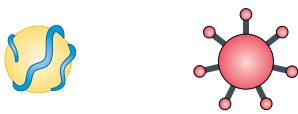

Lipoprotein-like Virus-like $\begin{array}{ll}\text { particles } & \text { pirus-like } \\ & \end{array}$ 
Such studies demonstrate the possibility of 'tuning' the NP corona to avoid unwanted ancillary effects. However, practical obstacles to this strategy must be surmounted; in addition to our lack of knowledge about the nano-bio interface, differences in patient physiology and dysregulated conditions in disease states could complicate efforts to model corona formation. Both the preformed corona strategy and the rational NP design strategy require vastly more sophisticated knowledge of corona dynamics and biological interfaces to be utilized effectively.

Biocompatible and biomimetic nanoparticles. The biocompatibility of a particle describes its ability to avoid triggering an adverse response from the surrounding host biology; as described, many of these adversities can arise from ancillary effects caused by unwanted interactions with a nanomaterial. For any clinical application, a high degree of biocompatibility is required. However, widely utilized clinical NP materials, such as PEG, may not be as biocompatible as previously believed; for example, multiple studies have found a high prevalence of anti-PEG antibodies in patient sera ${ }^{102,103}$, which suggests that PEG nanomaterial is actually fairly immunogenic. Therefore, it is important that we improve on existing nanomaterials. Basic aspects of pristine identity (physicochemistry, morphology, geometry, functional groups, density and so on) have a profound impact on NP biocompatibility, and thus can be adapted to help modulate the interactions with biology. For example, deformable disc-shaped polymeric particles have shown a favourable biocompatibility over rigid and spherical particles, perhaps because they mimic the size, shape and elasticity of erythrocytes ${ }^{104}$.

Beyond using artificial nanomaterials foreign to the host biology, another approach to biocompatibility is to incorporate biomaterials into the NP construction; this could be highly promising to mitigate adverse ancillary effects that arise from NPs, as endogenous materials are far less likely to provoke harmful responses. Biomimetic strategies for NPs can generally take one of two approaches. First, an NP can be 'disguised' with a biomimetic exterior, using natural biomolecules to cloak the foreign particle in seemingly native material (Fig. 6c). There are several plausible ways to implement this. A simple version of this strategy is similar to the corona pre-adsorption method described in 'Harnessing the corona architecture', except that the desired corona components would be covalently conjugated to the core NP, rather than adsorbed; in effect, this would allow the synthesis of a permanent biomimetic 'hard' corona that would not desorb in vivo. Another method involves surface conjugation of the NPs to cell-derived vesicles or platelets, or even to whole cells, to effectively 'hitchhike' the NPs and conceal them within the biological presence of the associated cell membrane, for example with NPs conjugated to erythrocytes ${ }^{105-107}$, although this might itself cause unintended NP-induced effects on the carrier erythrocytes and thus requires NP refinement ${ }^{108,109}$. Yet another adaptation of this strategy involves encapsulation of an NP inside cell-membrane-derived vesicles created from the host's own harvested cells (for example, erythrocytes ${ }^{110,111}$ or platelets ${ }^{112}$ ). From the outside, the NP appears to be indistinguishable from any other cell. In this case, the NP is covered by a cellular 'ghost', which would eventually degrade after uptake into the targeted tissue, freeing the core NP.

For the second avenue of biomimetic approach, a core NP can be constructed entirely from bioidentical molecules that are natively present in the host biology. The chemotherapeutic drug nab-paclitaxel (Abraxane) is an example of this biomimetic strategy, wherein the 'nanoparticle' is simply a human serum albumin protein used as a drug nanophore for paclitaxel delivery. This method could use any number of nanoscale biomaterials: lipoprotein-like particles, cell-membrane-derived vesicles, virus-like particles and so on. By using material identical to the host's own biology, such approaches could further reduce the unpredictable results that arise from the ancillary effects of foreign nanomaterials or from hypersensitivity reactions.

\section{Conclusions and perspectives}

Viewed through the prism of medicine, our overview of the current literature finds that the biological effects of NPs, the nanobiology, are not only eclectic, but also potentially a double-edged sword, which could contribute both to adverse effects and to novel mechanisms of therapeutic action. There are numerous pressing reasons to elucidate the nanobiology.

Novel indications for NPs as drugs. Nanomedicine promises the development of new therapeutic modalities that address a deficiency in the current state of medical care that small molecule drugs or other therapies simply cannot. NPs have largely been regarded as little more than a means to an end: a passive carrier meant only to unify a collection of functional modalities into a single macromolecule, created with a specific intended purpose and function. This applied science approach has resulted in a 'tunnel vision' of sorts, wherein only the biological effects elicited by the functional components of the NP are considered. This is a deficiency in the state of current research: as this Review shows, NPs alone can exhibit an eclectic array of ancillary effects, some of which may have therapeutic potential as nanomedicines, whereas others are detrimental or even potentially dangerous. Perhaps some of these effects could be exploited clinically as new treatments or could be exploited in rational design approaches to facilitate or synergize with a payload drug. At the very least, NPs should be re-evaluated as less-than-passive drug excipients which have effects independent of the payload. This is particularly important when evaluating the effect of a drug carried by NPs-how much of the effect is due to the particle versus the drug versus the combination of both? Often this is not considered appropriately.

Clinical suitability of NPs as drugs. Just as we lack adequate information to determine the best NP for a specific indication, we also lack sufficient information about nanobiology to determine when not to use a specific NP as a drug or vehicle. For example, many patient cohorts have special medical considerations: medication use, liver or kidney disease, pregnancy and so on. There is scant information to tell us what effects (if any) NPs might have in these populations. Nanomedicine contraindications are another knowledge gap that must be addressed. Furthermore, a more complete understanding of nanobiology will help us to avoid unnecessary errors of design, such as coupling a therapeutic payload to an NP carrier that actually elicits an antithetical ancillary effect and diminishes the therapeutic response.

Nanomaterial exposure outside of the clinic. NPs are not merely relevant only in the context of research or medicine. Some NP exposure is incidental and unavoidable due to the presence of nanomaterials in the environment. We know very little about the importance of naturally occurring NPs and how they have shaped and affected life on Earth. In addition, the prevalence of environmental NPs is ever increasing due to human industrial activity. NPs are widely used in consumer and industrial products and are inadvertently generated from basic human activities, such as the formation of fluorescent NPs in pizzas after heating ${ }^{113}$ or the emission of carbon NPs in vehicle exhausts ${ }^{114}$. The ecological impact of engineered NPs on natural flora and fauna is a concern, especially because of industrial-scale release of biopersistent NPs; for example, photosynthetic inhibition in aquatic microalgae and phytoplankton exposed to titanium dioxide NPs that originate from topical sunscreens in seawater ${ }^{115}$, or contamination of seafood with marine microplastics $^{116}$. NP contaminants raise concerns for both environmental conservation and for human health and welfare. It underlines the 
urgent scientific imperative to describe the continuum of interactions between NPs and biological systems at all levels of complexity.

Closing the knowledge gap. Even our basic knowledge of NPs and biology is in flux: for example, the EPR effect, long believed to be a passive process that arises from aberrant vasculature, may in fact be an active transport process, and therefore an effect of the nano-bio interface ${ }^{85}$. It is important to consider the reasons for our limited understanding of ancillary effects. Prior nanobiology studies are scattershot, limited and of questionable relevance: for example, many effects have been observed only in vitro, and the majority of in vivo studies have been performed in animal models, rather than in humans. Experimental NP dosages often are unattainable or not physiologically relevant. Furthermore, study findings might be applicable only to the very specific formulation of the NP used: many of the non-commercial NPs used probably were not synthesized under strict and standardized conditions; thus, even the true identity of the NP being investigated might be questionable. In this scenario, biological effects associated with the NP might actually be attributable to factors other than the NP itself, such as the presence of residual synthesis by-products due to inadequate purification ${ }^{117}$, the use of chemical dispersants to facilitate NP solubility ${ }^{118}$ or contamination with bacterial endotoxins ${ }^{119}$. Unfortunately, consistent systematic studies of NP-biological interactions have not been practical to date, due to experimental and model system heterogeneity, the lack of standards for information reporting in scientific publications and variations in NP manufacture, coupled with the rapid pace of innovation in this field. As a potential first step, Faria et al. proposed a minimum standard for information reporting in nanobiological studies ${ }^{120}$. Additional research standards along these lines would be helpful to make nanobiological research more methodical and systematic.

In addition to efforts to address these obstacles, it is crucial that we further our understanding with more comprehensive studies. Most NP studies do not consider ancillary biological effects at all, a short-sighted approach that should be rectified. The promise of nanomedicine cannot be fully realized if we do not understand how the engineered nanomaterials we create interact with the systems they are intended to regulate. This knowledge gap is the most pressing barrier to the emergence of nanomedicine.

Received: 11 September 2019; Accepted: 22 September 2021; Published online: 10 November 2021

\section{References}

1. Parhiz, H. et al. Unintended effects of drug carriers: big issues of small particles. Adv. Drug Deliv. Rev. 130, 90-112 (2018).

2. Monopoli, M. P., Aberg, C., Salvati, A. \& Dawson, K. A. Biomolecular coronas provide the biological identity of nanosized materials. Nat. Nanotechnol. 7, 779-786 (2012).

3. Kelly, P. M. et al. Mapping protein binding sites on the biomolecular corona of nanoparticles. Nat. Nanotechnol. 10, 472-479 (2015).

4. Nguyen, V. H. \& Lee, B. J. Protein corona: a new approach for nanomedicine design. Int. J. Nanomed. 12, 3137-3151 (2017).

5. Cox, A. et al. Evolution of nanoparticle protein corona across the blood-brain barrier. ACS Nano 12, 7292-7300 (2018)

6. Del Pilar Chantada-Vazquez, M. et al. Proteomic investigation on bio-corona of $\mathrm{Au}, \mathrm{Ag}$ and $\mathrm{Fe}$ nanoparticles for the discovery of triple negative breast cancer serum protein biomarkers. J. Proteom. 212, 103581 (2020).

7. Wang, F. et al. The biomolecular corona is retained during nanoparticle uptake and protects the cells from the damage induced by cationic nanoparticles until degraded in the lysosomes. Nanomedicine $\mathbf{9}$, 1159-1168 (2013).

8. Tenzer, S. et al. Rapid formation of plasma protein corona critically affects nanoparticle pathophysiology. Nat. Nanotechnol. 8, 772-781 (2013).

9. Foit, L. \& Thaxton, C. S. Synthetic high-density lipoprotein-like nanoparticles potently inhibit cell signaling and production of inflammatory mediators induced by lipopolysaccharide binding Toll-like receptor 4 . Biomaterials 100, 67-75 (2016).
10. Wan, S. et al. The 'sweet' side of the protein corona: effects of glycosylation on nanoparticle-cell interactions. ACS Nano 9, 2157-2166 (2015).

11. O'Connell, D. J. et al. Characterization of the bionano interface and mapping extrinsic interactions of the corona of nanomaterials. Nanoscale 7 15268-15276 (2015)

12. Lara, S. et al. Identification of receptor binding to the biomolecular corona of nanoparticles. ACS Nano 11, 1884-1893 (2017).

13. Gagner, J. E., Lopez, M. D., Dordick, J. S. \& Siegel, R. W. Effect of gold nanoparticle morphology on adsorbed protein structure and function. Biomaterials 32, 7241-7252 (2011).

14. Chaudhary, A., Khan, S., Gupta, A. \& Nandi, C. K. Effect of surface chemistry and morphology of gold nanoparticle on the structure and activity of common blood proteins. New J. Chem. 40, 4879-4883 (2016).

15. Trujillo-Alonso, V. et al. FDA-approved ferumoxytol displays anti-leukaemia efficacy against cells with low ferroportin levels. Nat. Nanotechnol. 14, 616-622 (2019).

16. Kim, S. E. et al. Ultrasmall nanoparticles induce ferroptosis in nutrient-deprived cancer cells and suppress tumour growth. Nat. Nanotechnol. 11, 977-985 (2016).

17. Daneman, R. \& Prat, A. The blood-brain barrier. Cold Spring Harb. Perspect. Biol. 7, a020412 (2015).

18. Jordan, C., Shuvaev, V. V., Bailey, M., Muzykantov, V. R. \& Dziubla, T. D. The role of carrier geometry in overcoming biological barriers to drug delivery. Curr. Pharm. Des. 22, 1259-1273 (2016).

19. Peng, F. et al. Nanoparticles promote in vivo breast cancer cell intravasation and extravasation by inducing endothelial leakiness. Nat. Nanotechnol. 14, 279-286 (2019).

20. Setyawati, M. I. et al. Titanium dioxide nanomaterials cause endothelial cell leakiness by disrupting the homophilic interaction of VE-cadherin. Nat. Commun. 4, 1673 (2013).

21. $\mathrm{Li}, \mathrm{C} . \mathrm{H}$. et al. Gold nanoparticles increase endothelial paracellular permeability by altering components of endothelial tight junctions, and increase blood-brain barrier permeability in mice. Toxicol. Sci. 148, 192-203 (2015).

22. Barui, A. K. et al. Zinc oxide nanoflowers make new blood vessels. Nanoscale 4, 7861-7869 (2012)

23. Shrivastava, S. et al. Characterization of antiplatelet properties of silver nanoparticles. ACS Nano 3, 1357-1364 (2009).

24. Ragaseema, V. M., Unnikrishnan, S., Kalliyana Krishnan, V. \& Krishnan, L. $\mathrm{K}$. The antithrombotic and antimicrobial properties of PEG-protected silver nanoparticle coated surfaces. Biomaterials 33, 3083-3092 (2012).

25. Jones, C. F. et al. Cationic PAMAM dendrimers aggressively initiate blood clot formation. ACS Nano 6, 9900-9910 (2012).

26. Bian, Y. et al. Titanium dioxide nanoparticles enhance thrombosis through triggering the phosphatidylserine exposure and procoagulant activation of red blood cells. Part. Fibre Toxicol. 18, 28 (2021).

27. Saikia, J. et al. Silica nanoparticle-endothelial interaction: uptake and effect on platelet adhesion under flow conditions. ACS Appl. Bio Mater. 1, 1620-1627 (2018).

28. Cao, J. et al. 'Adhesion and release' nanoparticle-mediated efficient inhibition of platelet activation disrupts endothelial barriers for enhanced drug delivery in tumors. Biomaterials 269, 120620 (2021).

29. $\mathrm{Yu}, \mathrm{X}$. et al. Changes of serum parameters of $\mathrm{TiO}_{2}$ nanoparticle-induced atherosclerosis in mice. J. Hazard. Mater. 280, 364-371 (2014).

30. Yan, Z. et al. Zinc oxide nanoparticle-induced atherosclerotic alterations in vitro and in vivo. Int. J. Nanomed. 12, 4433-4442 (2017).

31. Gilbert, J. A. et al. Current understanding of the human microbiome. Nat. Med. 24, 392-400 (2018).

32. Ruparelia, J. P., Chatterjee, A. K., Duttagupta, S. P. \& Mukherji, S. Strain specificity in antimicrobial activity of silver and copper nanoparticles. Acta Biomater. 4, 707-716 (2008)

33. Williams, K. et al. Effects of subchronic exposure of silver nanoparticles on intestinal microbiota and gut-associated immune responses in the ileum of Sprague-Dawley rats. Nanotoxicology 9, 279-289 (2015).

34. Javurek, A. B. et al. Gut dysbiosis and neurobehavioral alterations in rats exposed to silver nanoparticles. Sci. Rep. 7, 2822 (2017).

35. van den Brule, S. et al. Dietary silver nanoparticles can disturb the gut microbiota in mice. Part. Fibre Toxicol. 13, 38 (2016).

36. $\mathrm{Li}$, J. et al. Oral administration of rutile and anatase $\mathrm{TiO}_{2}$ nanoparticles shifts mouse gut microbiota structure. Nanoscale 10, 7736-7745 (2018).

37. Wang, L. et al. Impact of short-term exposure of AuNCs on the gut microbiota of BALB/c mice. J. Biomed. Nanotechnol. 15, 779-789 (2019).

38. Li, J. et al. $\mathrm{ZnO}$ nanoparticles act as supportive therapy in DSS-induced ulcerative colitis in mice by maintaining gut homeostasis and activating Nrf2 signaling. Sci. Rep. 7, 43126 (2017).

39. Gokulan, K., Bekele, A. Z., Drake, K. L. \& Khare, S. Responses of intestinal virome to silver nanoparticles: safety assessment by classical virology, whole-genome sequencing and bioinformatics approaches. Int. J. Nanomed. 13, 2857-2867 (2018) 
40. Alessandrini, F. et al. Pro-inflammatory versus immunomodulatory effects of silver nanoparticles in the lung: the critical role of dose, size and surface modification. Nanomaterials 7, 300 (2017).

41. Pushalkar, S. et al. The pancreatic cancer microbiome promotes oncogenesis by induction of innate and adaptive immune suppression. Cancer Discov. 8, 403-416 (2018).

42. Riquelme, E. et al. Tumor microbiome diversity and composition influence pancreatic cancer outcomes. Cell 178, 795-806 e712 (2019).

43. Balachandran, V. P. et al. Identification of unique neoantigen qualities in long-term survivors of pancreatic cancer. Nature 551, 512-516 (2017).

44. Aykut, B. et al. The fungal mycobiome promotes pancreatic oncogenesis via activation of MBL. Nature 574, 264-267 (2019)

45. Li, X., Aldayel, A. M. \& Cui, Z. Aluminum hydroxide nanoparticles show a stronger vaccine adjuvant activity than traditional aluminum hydroxide microparticles. J. Control. Release 173, 148-157 (2014).

46. Ruwona, T. B. et al. Toward understanding the mechanism underlying the strong adjuvant activity of aluminum salt nanoparticles. Vaccine 34, 3059-3067 (2016)

47. Khandhar, A. P. et al. Physicochemical structure of a polyacrylic acid stabilized nanoparticle alum (nanoalum) adjuvant governs $\mathrm{T}_{\mathrm{H}} 1$ differentiation of CD4 ${ }^{+}$T cells. Nanoscale 12, 2515-2523 (2020).

48. Swaminathan, G. et al. A novel lipid nanoparticle adjuvant significantly enhances $\mathrm{B}$ cell and $\mathrm{T}$ cell responses to sub-unit vaccine antigens. Vaccine 34, 110-119 (2016).

49. Thoryk, E. A. et al. Co-administration of lipid nanoparticles and sub-unit vaccine antigens is required for increase in antigen-specific immune responses in mice. Vaccines 4, 4 (2016).

50. Shirai, S. et al. Lipid nanoparticle acts as a potential adjuvant for influenza split vaccine without inducing inflammatory responses. Vaccines 8, 433 (2020).

51. La-Beck, N. M., Islam, M. R. \& Markiewski, M. M. Nanoparticle-induced complement activation: implications for cancer nanomedicine. Front. Immunol. 11, 603039 (2020).

52. Chen, F. et al. Complement proteins bind to nanoparticle protein corona and undergo dynamic exchange in vivo. Nat. Nanotechnol. 12, 387-393 (2017).

53. Banda, N. K. et al. Mechanisms of complement activation by dextran-coated superparamagnetic iron oxide (SPIO) nanoworms in mouse versus human serum. Part. Fibre Toxicol. 11, 64 (2014).

54. Wang, G. et al. Activation of human complement system by dextran-coated iron oxide nanoparticles is not affected by dextran/Fe ratio, hydroxyl modifications, and crosslinking. Front. Immunol. 7, 418 (2016).

55. Fornaguera, C. et al. Interactions of PLGA nanoparticles with blood components: protein adsorption, coagulation, activation of the complement system and hemolysis studies. Nanoscale 7, 6045-6058 (2015).

56. Inturi, S. et al. Modulatory role of surface coating of superparamagnetic iron oxide nanoworms in complement opsonization and leukocyte uptake. ACS Nano 9, 10758-10768 (2015).

57. Szebeni, J. et al. Liposome-induced complement activation and related cardiopulmonary distress in pigs: factors promoting reactogenicity of Doxil and AmBisome. Nanomedicine 8, 176-184 (2012).

58. Neun, B. W., Barenholz, Y., Szebeni, J. \& Dobrovolskai, M. A. Understanding the role of anti-PEG antibodies in the complement activation by Doxil in vitro. Molecules 23, 1700 (2018).

59. Hempel, J. C. et al. Distinct in vitro complement activation by various intravenous iron preparations. Am. J. Nephrol. 45, 49-59 (2017).

60. Mueller, S. N., Tian, S. \& DeSimone, J. M. Rapid and persistent delivery of antigen by lymph node targeting PRINT nanoparticle vaccine carrier to promote humoral immunity. Mol. Pharm. 12, 1356-1365 (2015).

61. Liu, Y. et al. Engineering biomaterial-associated complement activation to improve vaccine efficacy. Biomacromolecules 14, 3321-3328 (2013).

62. Ghendon, Y., Markushin, S., Akopova, I., Koptiaeva, I. \& Krivtsov, G. Chitosan as an adjuvant for poliovaccine. J. Med. Virol. 83, 847-852 (2011).

63. AbdelAllah, N. H. et al. Alginate-coated chitosan nanoparticles act as effective adjuvant for hepatitis A vaccine in mice. Int. J. Biol. Macromol. 152, 904-912 (2020).

64. Huaux, F. Emerging role of immunosuppression in diseases induced by micro- and nano-particles: time to revisit the exclusive inflammatory scenario. Front. Immunol. 9, 2364 (2018).

65. Getts, D. R. et al. Therapeutic inflammatory monocyte modulation using immune-modifying microparticles. Sci. Transl. Med. 6, 219 ra217 (2014).

66. Allen, R. P., Bolandparvaz, A., Ma, J. A., Manickam, V. A. \& Lewis, J. S. Latent, immunosuppressive nature of poly(lactic-co-glycolic acid) microparticles. ACS Biomater. Sci. Eng. 4, 900-918 (2018).

67. Cifuentes-Rius, A., Desai, A., Yuen, D., Johnston, A. P. R. \& Voelcker, N. H. Inducing immune tolerance with dendritic cell-targeting nanomedicines. Nat. Nanotechnol. 16, 37-46 (2021).

68. Smarr, C. B. et al. Biodegradable antigen-associated PLG nanoparticles tolerize $\mathrm{T}_{\mathrm{H}} 2$-mediated allergic airway inflammation pre- and postsensitization. Proc. Natl Acad. Sci. USA 113, 5059-5064 (2016).
69. Liu, Q. et al. Use of polymeric nanoparticle platform targeting the liver to induce Treg-mediated antigen-specific immune tolerance in a pulmonary allergen sensitization model. ACS Nano 13, 4778-4794 (2019).

70. Liu, Q. et al. Antigen- and epitope-delivering nanoparticles targeting liver induce comparable immunotolerance in allergic airway disease and anaphylaxis as nanoparticle-delivering pharmaceuticals. ACS Nano 15, 1608-1626 (2021)

71. Shah, S. et al. Optimizing PLG nanoparticle-peptide delivery platforms for transplantation tolerance using an allogeneic skin transplant model. Biomaterials 210, 70-82 (2019).

72. Bryant, J. et al. Nanoparticle delivery of donor antigens for transplant tolerance in allogeneic islet transplantation. Biomaterials 35, 8887-8894 (2014).

73. Wong, A. D., Ye, M., Ulmschneider, M. B. \& Searson, P. C. Quantitative analysis of the enhanced permeation and retention (EPR) effect. PLOS ONE 10, e0123461 (2015).

74. Ramanathan, R. K. et al. Correlation between ferumoxytol uptake in tumor lesions by MRI and response to nanoliposomal irinotecan in patients with advanced solid tumors: a pilot study. Clin. Cancer Res. 23, 3638-3648 (2017).

75. $\mathrm{Xu}$, J. et al. Multi-walled carbon nanotubes translocate into the pleural cavity and induce visceral mesothelial proliferation in rats. Cancer Sci. 103, 2045-2050 (2012).

76. Suzui, M. et al. Multiwalled carbon nanotubes intratracheally instilled into the rat lung induce development of pleural malignant mesothelioma and lung tumors. Cancer Sci. 107, 924-935 (2016).

77. Poland, C. A. et al. Carbon nanotubes introduced into the abdominal cavity of mice show asbestos-like pathogenicity in a pilot study. Nat. Nanotechnol. 3, 423-428 (2008)

78. Ryman-Rasmussen, J. P. et al. Inhaled carbon nanotubes reach the subpleural tissue in mice. Nat. Nanotechnol. 4, 747-751 (2009).

79. Carbon Black, Titanium Dioxide, and Talc (IARC, 2010).

80. Zanganeh, S. et al. Iron oxide nanoparticles inhibit tumour growth by inducing pro-inflammatory macrophage polarization in tumour tissues. Nat. Nanotechnol. 11, 986-994 (2016).

81. Plebanek, M. P., Bhaumik, D., Bryce, P. J. \& Thaxton, C. S. Scavenger receptor type B1 and lipoprotein nanoparticle inhibit myeloid-derived suppressor cells. Mol. Cancer Ther. 17, 686-697 (2018)

82. Rajan, R. et al. Liposome-induced immunosuppression and tumor growth is mediated by macrophages and mitigated by liposome-encapsulated alendronate. J. Control. Release 271, 139-148 (2018).

83. Saha, S. et al. Gold nanoparticle reprograms pancreatic tumor microenvironment and inhibits tumor growth. ACS Nano 10, 10636-10651 (2016).

84. Arvizo, R. R. et al. Inhibition of tumor growth and metastasis by a self-therapeutic nanoparticle. Proc. Natl Acad. Sci. USA 110, 6700-6705 (2013).

85. Sindhwani, S. et al. The entry of nanoparticles into solid tumours. Nat. Mater. 19, 566-575 (2020)

86. Su, S. \& Kang, P. M. Systemic review of biodegradable nanomaterials in nanomedicine. Nanomaterials 10, 656 (2020)

87. Longmire, M., Choyke, P. L. \& Kobayashi, H. Clearance properties of nano-sized particles and molecules as imaging agents: considerations and caveats. Nanomedicine 3, 703-717 (2008)

88. Du, B. J., Yu, M. X. \& Zheng, J. Transport and interactions of nanoparticles in the kidneys. Nat. Rev. Mater. 3, 358-374 (2018).

89. Benezra, M. et al. Multimodal silica nanoparticles are effective cancer-targeted probes in a model of human melanoma. J. Clin. Invest. 121, 2768-2780 (2011).

90. Phillips, E. et al. Clinical translation of an ultrasmall inorganic optical-PET imaging nanoparticle probe. Sci. Transl. Med. 6, 260ra149 (2014).

91. $\mathrm{Li}, \mathrm{Z}$. et al. Insight into the preformed albumin corona on in vitro and in vivo performances of albumin-selective nanoparticles. Asian J. Pharm. Sci. 14, 52-62 (2019).

92. Peng, Q. et al. Preformed albumin corona, a protective coating for nanoparticles based drug delivery system. Biomaterials 34, 8521-8530 (2013).

93. Peng, Q. et al. Enhanced biostability of nanoparticle-based drug delivery systems by albumin corona. Nanomedicine 10, 205-214 (2015).

94. Cao, H. et al. Albumin biomimetic nanocorona improves tumor targeting and penetration for synergistic therapy of metastatic breast cancer. $A d v$. Funct. Mater. 27, 1605679 (2017).

95. Bargheer, D. et al. The fate of a designed protein corona on nanoparticles in vitro and in vivo. Beilstein J. Nanotechnol. 6, 36-46 (2015).

96. Ban, Z. et al. Machine learning predicts the functional composition of the protein corona and the cellular recognition of nanoparticles. Proc. Natl Acad. Sci. USA 117, 10492-10499 (2020).

97. Walkey, C. D. et al. Protein corona fingerprinting predicts the cellular interaction of gold and silver nanoparticles. ACS Nano 8, 2439-2455 (2014). 
98. Palchetti, S. et al. Protein corona fingerprints of liposomes: new opportunities for targeted drug delivery and early detection in pancreatic cancer. Pharmaceutics 11, 31 (2019).

99. Coty, J. B., Eleamen Oliveira, E. \& Vauthier, C. Tuning complement activation and pathway through controlled molecular architecture of dextran chains in nanoparticle corona. Int. J. Pharm. 532, 769-778 (2017)

100. Santi, M. et al. Rational design of a transferrin-binding peptide sequence tailored to targeted nanoparticle internalization. Bioconjug. Chem. 28, 471-480 (2017).

101. Vincent, M. P. et al. Surface chemistry-mediated modulation of adsorbed albumin folding state specifies nanocarrier clearance by distinct macrophage subsets. Nat. Commun. 12, 648 (2021).

102. Yang, Q. et al. Analysis of pre-existing IgG and IgM antibodies against polyethylene glycol (PEG) in the general population. Anal. Chem. 88, 11804-11812 (2016).

103. Chen, B. M. et al. Measurement of pre-existing IgG and IgM antibodies against polyethylene glycol in healthy individuals. Anal. Chem. 88, 10661-10666 (2016).

104. Palange, A. L., Palomba, R., Rizzuti, I. F., Ferreira, M. \& Decuzzi, P. Deformable discoidal polymeric nanoconstructs for the precise delivery of therapeutic and imaging agents. Mol. Ther. 25, 1514-1521 (2017).

105. Wibroe, P. P. et al. Bypassing adverse injection reactions to nanoparticles through shape modification and attachment to erythrocytes. Nat. Nanotechnol. 12, 589-594 (2017).

106. Brenner, J. S. et al. Red blood cell-hitchhiking boosts delivery of nanocarriers to chosen organs by orders of magnitude. Nat. Commun. 9, 2684 (2018).

107. Zhao, Z., Ukidve, A., Gao, Y., Kim, J. \& Mitragotri, S. Erythrocyte leveraged chemotherapy (ELeCt): nanoparticle assembly on erythrocyte surface to combat lung metastasis. Sci. Adv. 5, eaax9250 (2019).

108. Pan, D. C. et al. Nanoparticle properties modulate their attachment and effect on carrier red blood cells. Sci. Rep. 8, 1615 (2018).

109. Pan, D. et al. The effect of polymeric nanoparticles on biocompatibility of carrier red blood cells. PLoS ONE 11, e0152074 (2016).

110. Piao, J. G. et al. Erythrocyte membrane is an alternative coating to polyethylene glycol for prolonging the circulation lifetime of gold nanocages for photothermal therapy. ACS Nano 8, 10414-10425 (2014).

111. Hu, C. M. J. et al. Erythrocyte membrane-camouflaged polymeric nanoparticles as a biomimetic delivery platform. Proc. Natl Acad. Sci. USA 108, 10980-10985 (2011).

112. $\mathrm{Hu}, \mathrm{C}$. M. et al. Nanoparticle biointerfacing by platelet membrane cloaking. Nature 526, 118-121 (2015).
113. Cong, S. et al. Fluorescent nanoparticles in the popular pizza: properties, biodistribution and cytotoxicity. Food Funct. 10, 2408-2416 (2019).

114. Holmen, B. A. \& Ayala, A. Ultrafine PM emissions from natural gas, oxidation-catalyst diesel, and particle-trap diesel heavy-duty transit buses. Environ. Sci. Technol. 36, 5041-5050 (2002).

115. Sendra, M. et al. Effects of $\mathrm{TiO}_{2}$ nanoparticles and sunscreens on coastal marine microalgae: ultraviolet radiation is key variable for toxicity assessment. Environ. Int. 98, 62-68 (2017).

116. Smith, M., Love, D. C., Rochman, C. M. \& Neff, R. A. Microplastics in seafood and the implications for human health. Curr. Environ. Health Rep. 5, 375-386 (2018)

117. Henry, T. B., Petersen, E. J. \& Compton, R. N. Aqueous fullerene aggregates $\left(n \mathrm{C}_{60}\right)$ generate minimal reactive oxygen species and are of low toxicity in fish: a revision of previous reports. Curr. Opin. Biotechnol. 22, 533-537 (2011).

118. Jo, M. R. et al. Effects of zinc oxide nanoparticle dispersants on cytotoxicity and cellular uptake. Mol. Cellular Toxicol. 12, 281-288 (2016).

119. Li, Y. \& Boraschi, D. Endotoxin contamination: a key element in the interpretation of nanosafety studies. Nanomedicine 11, 269-287 (2016).

120. Faria, M. et al. Minimum information reporting in bio-nano experimental literature. Nat. Nanotechnol. 13, 777-785 (2018).

121. Tarangelo, A., \& Dixon, S. J. An iron age for cancer therapy. Nat. Nanotechnol. 11, 921-922 (2016)

\section{Acknowledgements}

We thank the Ella Maru Studio for assistance in the graphic design of figures in this Review and acknowledge partial financial support from NIH (5R01CA215700-05 and 5R01CA218615-04) to JG

\section{Competing interests}

The authors declare no competing interests.

\section{Additional information}

Correspondence should be addressed to Jan Grimm.

Peer review information Nature Nanotechnology thanks Paolo Decuzzi and the other, anonymous, reviewer(s) for their contribution to the peer review of this work.

Reprints and permissions information is available at www.nature.com/reprints. Publisher's note Springer Nature remains neutral with regard to jurisdictional claims in published maps and institutional affiliations.

(c) Springer Nature Limited 2021 